\title{
High-throughput approaches for precision medicine in high-grade serous ovarian cancer
}

\author{
Meinusha Govindarajan' ${ }^{1}$ Christoph Wohlmuth ${ }^{2,3}$, Matthew Waas ${ }^{4}$, Marcus Q. Bernardini ${ }^{2,5^{*}}$ \\ and Thomas Kislinger ${ }^{1,4^{*}}$ (D)
}

\begin{abstract}
High-grade serous carcinoma (HGSC) is the most prevalent and aggressive subtype of ovarian cancer. The large degree of clinical heterogeneity within HGSC has justified deviations from the traditional one-size-fits-all clinical management approach. However, the majority of HGSC patients still relapse with chemo-resistant cancer and eventually succumb to their disease, evidence that further work is needed to improve patient outcomes. Advancements in high-throughput technologies have enabled novel insights into biological complexity, offering a large potential for informing precision medicine efforts. Here, we review the current landscape of clinical management for HGSC and highlight applications of high-throughput biological approaches for molecular subtyping and the discovery of putative blood-based biomarkers and novel therapeutic targets. Additionally, we present recent improvements in model systems and discuss how their intersection with high-throughput platforms and technological advancements is positioned to accelerate the realization of precision medicine in HGSC.
\end{abstract}

Keywords: High-grade serous carcinoma, High-throughput technology, Liquid biopsies, Therapeutic targets, Molecular subtypes

\section{Background}

The American Cancer Society estimates that in 2020, 21,750 women will be newly diagnosed with ovarian cancer in the USA and 13,940 will die from the disease [1]. Epithelial ovarian cancer (EOC) represents the fifth most common cause of cancer death overall and is the leading cause of death from gynecologic malignancies in the USA, Canada and Europe [1-3]. EOC is a heterogeneous disease with different types of histologies, molecular and microenvironmental features [4]. Histologically, EOC is traditionally classified into five major subtypes: high-grade serous (HGSC), low-grade serous (LGSC), clear cell, endometrioid and mucinous

\footnotetext{
*Correspondence: Marcus.Bernardini@uhn.ca; thomas.kislinger@utoronto.ca ${ }^{1}$ Department of Medical Biophysics, University of Toronto, Toronto, Canada

2 Division of Gynecologic Oncology, Princess Margaret Cancer Centre, University Health Network, Toronto, Canada

Full list of author information is available at the end of the article
}

ovarian cancer [4]. A more recent classification model categorizes EOC into type I and II tumors, where LGSC, endometrioid, mucinous and clear cell carcinomas are classified as type I [5-7]. These neoplasms typically present as large, unilateral, cystic tumors and clinically tend to behave in an indolent fashion [7]. Genetically, type I cancers are characterized by minor chromosomal instability and may harbor BRAF, KRAS and PTEN mutations. Type II cancers, on the other hand, comprise of HGSC, which account for the vast majority of all EOCs, carcinosarcomas and undifferentiated carcinomas. HGSCs have a high degree of genetic instability and are characterized by the presence of acquired or inherited mutations in different DNA repair pathways including TP53, BRCA1/2 and other defects in homologous recombination repair genes $[4,5,7]$. Recent evidence suggests that HGSC originates from the fimbriae of the fallopian tube secondarily involving the ovary and peritoneum [8]. original author(s) and the source, provide a link to the Creative Commons licence, and indicate if changes were made. The images or other third party material in this article are included in the article's Creative Commons licence, unless indicated otherwise in a credit line to the material. If material is not included in the article's Creative Commons licence and your intended use is not permitted by statutory regulation or exceeds the permitted use, you will need to obtain permission directly from the copyright holder. To view a copy of this licence, visit http://creativecommons.org/licenses/by/4.0/. The Creative Commons Public Domain Dedication waiver (http://creativeco mmons.org/publicdomain/zero/1.0/) applies to the data made available in this article, unless otherwise stated in a credit line to the data. 
Early-stage disease is typically asymptomatic, and currently there are no proven screening strategies for HGSC that reduce mortality $[9,10]$. The tumor volume in the ovaries is substantially less than that of type I tumors, and $80 \%$ of HGSCs are diagnosed at advanced disease stages [7, 11]. Even in advanced HGSC, symptoms are nonspecific and include back pain, fatigue, bloating, constipation, abdominal pain, change in bowel function, urinary symptoms and weight loss [12]. The initial diagnostic work-up includes a pelvic ultrasound or computed tomography (CT) and (CA125) assessment [13]. Magnetic resonance imaging may be used to further stratify pelvic masses, and a CT of the thorax, abdomen and pelvis is performed for staging purposes. The standard of care treatment for HGSC is primary debulking surgery (PDS) to no visible residual disease with adjuvant platinum-based chemotherapy. Two randomized trials comparing PDS and chemotherapy with neoadjuvant chemotherapy followed by interval debulking surgery showed similar survival, but both studies had minimal residual disease and survival rates in both study arms [13-15]. Despite recent advances, approximately $70 \%$ of EOCs recur and the 5-year survival rate for metastatic disease remains poor at $30 \%[1,16]$.

Precision medicine refers to the notion of tailoring clinical management of diseases to account for patient heterogeneity. Although it is well known that EOC comprises several pathologically distinct diseases, the current standard of care is to generally manage these subtypes as a single entity. Molecular screening has revealed a vast degree of variability within the HGSC subtype itself [17]. This is reflected in the array of clinical outcomes as not all patients respond to conventional therapies. This underlying complexity also makes it unlikely that a single tumor marker will be effective for all patients. The use of poly (ADP-ribose) polymerase (PARP) inhibitors for patients with $B R C A 1 / 2$ mutations is an example of the shift toward precision medicine in HGSC; however, additional work is still necessary. Biomarkers that are reflective of tumor burden and therapies which target specific tumor characteristics are needed to improve patient outcomes. Advancements in high-throughput biological techniques have provided new opportunities for the discovery of biomarkers and therapeutic targets. These platforms allow for the simultaneous profiling of thousands of molecules and the subsequent generation of a wealth of biological data. Together with large cohorts of wellannotated patient samples and improved model systems, these approaches have facilitated novel insights into biological heterogeneity at an unprecedented scale. In this review, we provide an overview of how high-throughput approaches have contributed to the molecular profiling of patient heterogeneity within HGSC and highlight the utility of these technologies in the discovery of putative blood-based biomarkers and therapeutic targets as a step toward enabling precision medicine as a reality for all HGSC patients (Fig. 1). We also discuss the complementary role of HGSC experimental models in advancing these discoveries.

\section{Molecular tumor profiling of HGSC}

High-throughput molecular profiling of tumor samples has been used to gain insights into the biological aberrations underlying the pathogenesis of HGSC. The largest study in mapping the molecular features of HGSC was conducted by The Cancer Genome Atlas (TCGA) network, where 489 tumor samples were subjected to genomic and transcriptomic analyses [17]. Exome sequencing detected TP53 mutations in 96\% of tumors. Interestingly, subsequent histological analysis of the TP53 wild-type tumors in this cohort revealed differences in morphological features indicating that these tissues were not truly HGSC tumors [18], suggesting the proportion of TP53 mutations to be even higher than reported. This finding is consistent with other reports of ubiquitous TP53 mutations in HGSC [19]. Serous tubal intraepithelial carcinomas (STIC) (the precursor lesion of HGSC) and 'p53-signature lesions' (the hypothesized precursor of STIC) in the fallopian tube have been shown to share identical TP53 mutations to HGSC, signifying that TP53 mutations develop early in the HGSC carcinogenic process [20]. Germline and somatic mutations in BRCA1 and BRCA2 are the next most prevalent mutations in HGSC, cumulatively present in $22 \%$ of the TCGA cohort [16]. Seven other significantly mutated genes were identified albeit only in 2-6\% of cases, demonstrating a limited mutational landscape in HGSC. In contrast, HGSC exhibits a high degree of chromosomal instability evident by extensive copy number alterations (CNAs) in each tumor and the identification of 113 significantly recurrent CNAs throughout the entire cohort [17]. The TCGA study also revealed that half of HGSC tumors had genomic and/or epigenetic deficiencies in homologous recombination, further underscoring the role of erroneous DNA repair mechanisms in HGSC pathogenesis [17]. Indeed, homologous repair deficiency (HRD) is a crucial determinant of platinum sensitivity in HGSC [21]. Other frequently altered pathways in HGSC include RB1, PI3K/ RAS, NOTCH and FOXM1 [17]. In an attempt to deconvolute this vast genomic heterogeneity, Macintyre et al. [22] have recently identified seven copy number signatures in HGSC, some of which were found to be associated with previously mentioned mutations, aberrant 


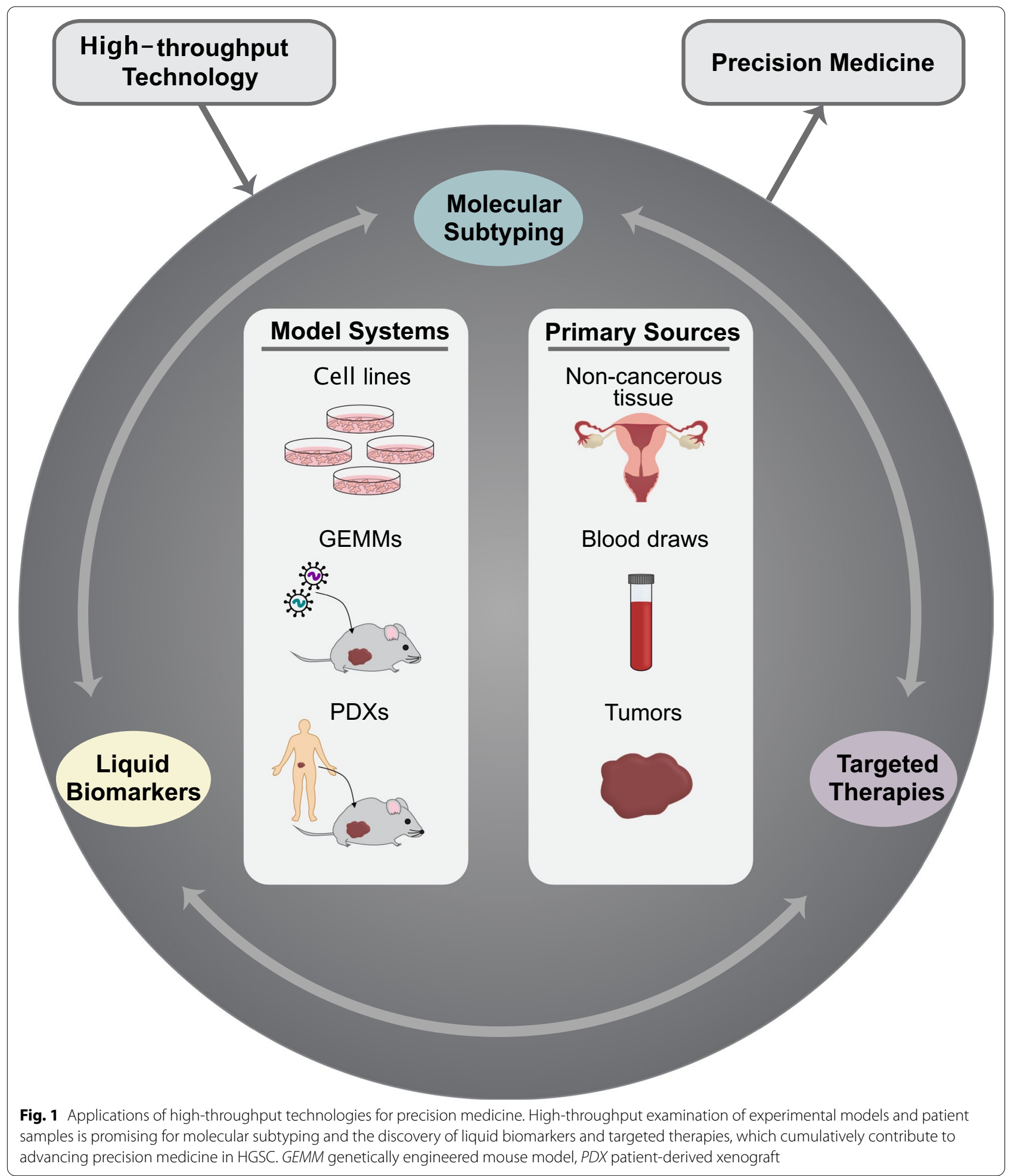

pathways and survival outcomes, yet larger studies are still required to validate these associations.

The profiling of mRNA expression in HGSC tumors has identified four overlapping transcriptional subtypes of
HGSC: C1-mesenchmyal, C2-immunoreactive, C4differentiated and C5-proliferative [17, 23]. Independent studies have identified prognostic implications associated with these subtypes in which the immunoreactive subtype 
exhibited improved survival outcomes, whereas the mesenchymal and proliferative subtypes demonstrated the worst overall survivals $[24,25]$. Building on these consistent findings, Leong et al. [26] have identified a gene signature consisting of 39 differentially expressed genes for classification of these subtypes. The Clinical Proteomic Tumor Analysis Consortium (CPTAC) analyzed the global proteomes of 169 HGSC tumors from the TCGA cohort [27]. Clustering of tumors based on protein abundance revealed five subtypes, four of which demonstrated a clear resemblance to the classical transcriptomic subtypes and one novel subtype classified as stromal [27]. Integration of proteomic and CNA data revealed that proteins associated with multiple CNAs were enriched in cell invasion/migration and immune processes, suggesting there is a functional convergence of the high degree of chromosomal instability [27]. The low overall correlation between mRNA expression and protein expression in this investigation highlights the importance of multi-omic profiling to achieve a comprehensive understanding of molecular alterations underlying HGSC [27].

Aside from delineating molecular heterogeneity between patients, high-throughput tumor profiling can also be used to elucidate the diversity within a tumor. Deconvolution of bulk HGSC transcriptional data has revealed that individual tumors often display multiple subtype signatures [25], accentuating the additional layer of molecular complexity offered by intratumor heterogeneity. Albeit on a small scale, recent efforts in multiregion tumor profiling of HGSC tumors have uncovered intratumor molecular heterogeneity in both a spatial manner and temporal manner [26, 2831]. Although larger investigations are warranted to extend the generalizability of these data, these studies highlight the susceptibility of bulk subtypes to sampling bias and the potential confounding role of stromal components in tumor profiling. Additionally, in a disease characterized by extensive intraperitoneal dissemination, multiregion molecular profiling of primary tumors and metastases can be of value for discerning the biology underlying HGSC progression [32-34]. Despite the loss of spatial microenvironment context, single-cell technologies can also provide insights into intratumor heterogeneity [35, 36]. Further large-scale studies using these emerging approaches may shed light into how intratumor heterogeneity manifests in clinical outcomes such as therapeutic resistance. Overall, the spectrum of molecular differences within HGSC underscores the significance in using high-throughput approaches to further understand the biological abnormalities and translate these findings into novel biomarkers and targeted therapies.

\section{Blood-based biomarkers for HGSC}

A biomarker is a measurable feature that is reflective of biological processes and can provide information regarding the disease state of an individual. Cancer biomarkers are used for various purposes throughout the course of disease progression, including assessing the likelihood of developing cancer, diagnosing malignancies, determining prognosis, predicting patient responses to specific therapies and monitoring residual disease posttreatment and during remission. In contrast to directly examining tumor tissue, liquid biopsies can facilitate minimally invasive tumor assessments to guide clinical decisions. Blood is an attractive biological fluid for biomarkers in clinical practice due to the standardized collection procedures and abundant availability. In this section, we briefly review the current landscape of HGSC blood-based biomarkers and discuss the utility of high-throughput approaches in the discovery of novel biomarkers to help improve clinical management of HGSC.

\section{Liquid biopsies in clinical practice}

In the context of HGSC, and EOC in general, serum biomarkers are currently used for the differential diagnosis of a pelvic mass prior to surgery, monitoring response to treatment and detecting recurrent disease. Although definitive diagnosis of EOC currently requires histological examination, differential diagnosis of a pelvic mass determines preoperative referral [37]. This is crucial as optimal tumor resection and subsequent improved outcomes are more likely when surgical management for EOC is performed by gynecological oncologists rather than general surgeons or gynecologists [38]. In addition to determining treatment efficacy and prognosis following therapy, accurate markers of treatment response are also used to evaluate novel therapies in clinical trials [39]. Considering the high rates of HGSC relapse, early detection of recurrent disease is imperative for appropriate timing of therapies to improve survival [40].

\section{Cancer antigen 125}

CA125 is a large membrane glycoprotein encoded by the gene MUC16 and was identified as a tumor marker for EOC in 1983 [41]. Significant expression of CA125 is observed in $85 \%$ of serous, $65 \%$ of endometrioid, $40 \%$ of clear cell, $36 \%$ of undifferentiated and $12 \%$ of mucinous ovarian cancers, highlighting the lack of utility of CA125 in some EOC subtypes [42]. Despite being the most widely used biomarker for EOC, CA125 offers limited value as a diagnostic test. Serum concentrations of CA125 are elevated in $90 \%$ of advanced-stage EOCs 
and less than $50 \%$ of early-stage EOCs, resulting in a low sensitivity for detecting early-stage disease [43]. Furthermore, serum CA125 abundance has a low specificity for EOC as levels can be increased due to multiple benign gynecological and medical conditions including endometriosis and pregnancy [44]. The low specificity is especially manifest in premenopausal women who are at an increased risk of many of these other conditions [45]. Given the limitations of CA125 as a stand-alone diagnostic marker, the Risk of Malignancy Index (RMI) [46] and the International Ovarian Tumor Analysis (IOTA) Adnex model [47] were developed to integrate serum CA125 levels, ultrasound criteria and demographics, resulting in improved specificity and sensitivity for differential diagnosis of pelvic masses prior to surgery. When evaluated as a potential screening test, both the UKCTOCS study [9] and the PLCO trial [48] demonstrated that serum testing of CA125 alone or combined with transvaginal ultrasound imaging did not reduce mortality due to EOC and resulted in an increase in unnecessary invasive procedures associated with complications, underlining the clinical consequences of low specificity.

Nevertheless, CA125 offers clinical utility when evaluating treatment response and monitoring remission. A decrease in serum CA125 is indicative of treatment response, whereas a persistence of abnormally elevated CA125 or increases may suggest treatment resistance and/or residual disease [39]. Many post-treatment surveillance protocols include serial measurements of CA125, as rising serum CA125 is strongly predictive of disease recurrence [49-51]. A rise in CA125 concentration has been shown to precede clinical detection of recurrent disease by at least three to five months $[49,52,53]$. However, up to half of patients within the normal limits of CA125 during remission are found to have small volumes of disease during a second-look surgery $[54,55]$. Hence, despite being the earliest sign of recurrence currently available, CA125 is not optimally sensitive for detecting recurrence in all patients.

\section{Human epididymis protein 4}

Human epididymis protein 4 (HE4), encoded by the gene $W F D C 2$, is a secreted glycoprotein that is overexpressed in serous and endometrioid ovarian cancers [56]. Hellstrom et al. [57] initially determined that serum HE4 was comparable to CA125 for distinguishing between patients with advanced-stage disease and healthy controls. Subsequent studies have produced conflicting reports regarding the sensitivity of HE4 compared to CA125 as a diagnostic test, yet there is a consensus that HE4 is more specific than CA125, especially in premenopausal women [58-61]. This superiority is likely due to serum levels of HE4 being less influenced by other gynecological disorders such as endometriosis [62]. Serum HE4 is also elevated in at least a third of patients who do not demonstrate elevated serum CA125 levels, signifying a role for complementary markers in diagnostics [63]. Serum HE4 is currently approved to be used as a tumor marker for monitoring disease progression and recurrence. A study evaluating serum levels of HE4 and CA125 prior to surgery for suspicious recurrent EOC found HE4 to be more sensitive and specific than CA125 [64]. In a pilot study, Anastasi et al. [65] found that a rise in serum HE4 preceded elevated serum CA125 five to eight months in five out of eight patients with recurrent disease. Preliminary studies have also revealed that HE4 elevation can detect recurrence in a subset of EOC patients that do not present with increased serum CA125 [66, 67]. The combination of both markers resulted in a higher sensitivity and specificity in detecting recurrence than either marker alone [68], but data from larger prospective trials, including potential benefits in survival, are still pending.

\section{Multimarker assays}

In response to the promising data regarding the complementary potential between CA125 and HE4 as diagnostic markers, the Risk of Malignancy Algorithm (ROMA) was developed and is currently approved for differential diagnosis [69]. ROMA combines serum measurements of CA125 and HE4 and uses two different logarithmic regression models based on menopausal status to determine the likelihood of malignancy in women who are having surgery for pelvic masses [70]. A meta-analysis comparing ROMA, HE4 and CA125 revealed that ROMA demonstrated the greatest sensitivity and HE4 exhibited the highest specificity for differential diagnosis, although these differences were not statistically significant [71]. In a direct comparison, ROMA was found to be more sensitive than RMI with similar specificities [72], yet both assays demonstrated low sensitivity for early-stage disease [73]. OVA1 is a biomarker panel of five proteins used for the differential diagnosis of pelvic masses prior to surgery. The test consists of immunoassays for two upregulated proteins (CA125, beta 2 microglobulin) and three down-regulated proteins (transferrin, transthyretin, apolipoprotein A1) in serum [74]. An algorithm is used to integrate the measurements of each marker to generate an ovarian malignancy risk score ranging from $0-10$. The threshold for risk of malignancy is dependent on menopausal status. Many prospective studies comparing the performance of OVA1 to CA125 have reported higher sensitivity than CA125, especially for early-stage disease, yet lower specificity [75-77]. There have been no 
direct comparisons between OVA1 and ROMA to date. Overa is a second-generation multivariate assay which was intended to overcome the low specificity of OVA1 [78]. Overa uses serum measurements of CA125, HE4, apolipoprotein A1, transferrin and follicle-stimulating hormone to assess the likelihood of malignancy in women who will undergo surgery for a pelvic mass. The incorporation of follicle-stimulating hormone eliminates the need for assessing menopausal status as in OVA1. Overa was designed and validated using the same study population as OVA1, thus allowing for direct comparisons between the two assays. Indeed, Overa demonstrated an improved specificity and similar sensitivity to OVA1 [78].

\section{Germline BRCA1/2 mutations}

Germline deficiencies in $B R C A 1 / 2$ are the strongest genetic risk factors for nonmucinous EOC [79]. The cumulative risk of developing ovarian cancer in BRCA1 and $B R C A 2$ carriers ranges from 40 to $59 \%$ and 16 to $18 \%$, respectively [80-82]. As such, genetic counseling and genetic testing can be suggested for patients with familial history of breast, ovarian, pancreatic or prostate cancer to identify those who are at an elevated risk [83] and will likely benefit from preventative measures such as risk-reducing bilateral salpingo-oophorectomies [84, 85]. In addition, germline and somatic BRCA1/2 mutations are considered predictive biomarkers due to strong associations with favorable outcomes following both platinum-based chemotherapy $[86,87]$ and maintenance therapy with PARP inhibitors $[88,89]$. PARP inhibitors have also been approved for usage as monotherapy in $B R C A 1 / 2$-deficient women with recurrent disease [90, 91]. Hence, genetic testing for $B R C A 1 / 2$ mutations is recommended for all newly diagnosed ovarian cancer patients to aid in therapy selection and determining cancer risk for family [83].

\section{Emerging high-throughput biomarker discovery approaches}

Though blood is an attractive source of biomarkers, limitations in the throughput of highly sensitive molecular measurements have been a challenge, especially for heterogeneous diseases such as HSGC. Advancements in 'omics' approaches have enabled the ability to characterize and evaluate various classes of circulating molecules as potential blood-based biomarkers (Fig. 2). A biomarker discovery pipeline is typically initiated with a discovery phase in which large-scale comparative profiling experiments of blood, tumor tissue or model systems are used to generate a list of candidate markers. Following the discovery phase, targeted methods of quantification are often applied to validate candidate markers in clinical samples [92]. Here, we discuss the various classes of molecules and types of discovery approaches which have been applied to HSGC biomarker discovery.






\section{Circulating tumor DNA}

Circulating cell-free DNA (cfDNA) are short fragments of DNA released into the bloodstream from apoptotic or necrotic cells [93]. The quantification of total cfDNA has revealed that EOC patients have elevated levels of cfDNA compared to healthy controls and patients with benign disease [94-96]. However, evaluation of cfDNA abundance is not a direct measure of tumor burden as DNA fragments are also released by noncancerous cells. The fraction of cfDNA that originated from tumor cells, termed circulating tumor DNA (ctDNA), can be distinguished by the presence of cancer-specific alterations [97]. Given the low abundance of ctDNA compared to cfDNA, highly sensitive approaches such as digital polymerase chain reaction (PCR) and targeted next-generation sequencing (NGS) are used to detect cancer-specific modifications. Since TP53 mutations are ubiquitous in HGSC, detection of TP53 mutants in cfDNA has been preferentially used when investigating ctDNA as a biomarker [98-101]. Parkinson et al. [98] analyzed TP53 mutations in longitudinal plasma samples from HGSC patients undergoing treatment to evaluate the value of ctDNA in determining prognosis and response to treatment. This study revealed that the abundance of TP53 mutant ctDNA fractions prior to treatment significantly correlated with volumetric measurements of tumors from CT images, unlike CA125, and that a decrease of $>60 \%$ of TP53 mutant ctDNA fractions following treatment was a predictor of timeto-progression. Christie et al. [102] recently investigated whether reversal of germline $B R C A 1 / 2$ mutations can be detected in ctDNA, as this molecular alteration is known to correspond with acquired chemo-resistance. Reversion mutations were detected in the plasma of three out of five patients with reversion mutations observed in tumor samples, all of whom were resistant to platinumbased therapy or PARP inhibitors. The authors noted that detection of reversal mutations was associated with the fraction of ctDNA out of total cfDNA, measured by the presence of TP53 mutant alleles. Certainly, a limiting factor of utilizing ctDNA as a tumor marker is that current detection strategies may not be sensitive enough to detect rare mutants in early-stage disease where the ctDNA fraction is low [103].

In addition to investigating specific genes, examination of genome-wide chromosomal aberrations in ctDNA can be promising for the discovery of novel tumor markers. Harris et al. [104] used whole-genome sequencing to characterize genomic rearrangements in primary tumors of HGSC patients and investigated whether patient-specific aberrant chromosomal junctions could be detected in plasma. ctDNA with patient-specific chromosomal alterations was detected in pre-surgically drawn plasma samples for eight out of ten patients. Postsurgical detection of ctDNA was specific to the only three patients with clinically documented residual disease, suggesting a potential for personalized markers of tumor burden [104]. As changes in DNA methylation and chromatin remodeling play a role in tumor biology, the rise of epigenetic technologies (e.g., methylation profiling) is also promising for the use of ctDNA as blood-based tumor markers [105]. In the context of HGSC, Widschwednter et al. [106] identified aberrant methylation signatures in tumor tissues and developed a three-marker DNA methylation panel for ctDNA that was able to discriminate patients from healthy women or women with benign masses. The panel was also shown to better distinguish between platinum responders and nonresponders than CA125.

\section{microRNAs}

microRNAs (miRNAs) are a class of short (19-25 nucleotides) noncoding RNAs that are involved in gene regulation. miRNAs can function as oncogenes or tumor suppressors depending on cellular context, and expression has been shown to be deregulated in several cancers [107]. miRNAs are actively secreted from cells by binding to protein complexes or by being packaged into extracellular vesicles, thus providing protection from RNAse digestion and degradation in various extreme conditions (e.g., high temperatures, severe $\mathrm{pH}$ and multiple freeze-thaw cycles) [108, 109]. The stability of miRNAs in blood renders them as attractive molecules for tumor markers in liquid biopsies. In large-scale miRNA biomarker discovery experiments, high-throughput qRT-PCR panels, microarrays and more recently, NGS, can be used for profiling miRNAs in patient samples [110-112]. Todeschini et al. [110] used microarrays to profile miRNA expression in sera from HGSC patients and healthy controls. The differentially expressed miRNAs were then quantified in an independent cohort from which a single miRNA that demonstrated the greatest ability in discriminating HGSC patients from controls was identified as a putative diagnostic biomarker. Shah et al. [111] used qRT-PCR panels for serum miRNA profiling and demonstrated that combining measurements of circulating miRNAs and CA125 can be predictive of surgical resection outcomes for women with HGSC, suggesting value for circulating miRNAs as prognostic markers.

\section{Proteins}

Proteins are the primary functional elements of most biological processes, and thus, protein expression is often deregulated in disease states. Mass spectrometry (MS) is a powerful approach for protein measurement as current 
MS-based proteomic experiments can detect thousands of proteins in a single sample. MS has already proven to be fruitful in EOC biomarker discovery as the four markers in OVA1 (excluding CA125) were discovered using MS-based approaches [74]. In the studies that led to the development of OVA1, seven protein candidates were identified in the original discovery phase, yet verification of candidates was limited to only those proteins which had existing immunoassays [113, 114]. Though this approach is advantageous for faster clinical adoption, antibody availability can pose as a bottleneck for validation of candidate markers in biomarker discovery pipelines. Targeted MS approaches such as multiple reaction monitoring (MRM) and the more recently developed parallel reaction monitoring (PRM) can enable high-throughput robust quantification independent of antibody availability, thus circumventing the need for antibody development during biomarker discovery.

Detection of blood-based protein markers is challenging due to the large dynamic range and high sample complexity of serum/plasma. Considering that the 22 most abundant proteins in plasma account for $99 \%$ of the total mass of protein [115], detection of low-abundance proteins, often the most promising proteins for biomarker candidates, is hindered. Several preanalytical workflows have been developed to overcome this complexity, including the depletion of high-abundance proteins, sample fractionation and/or the enrichment for sub-proteomes [116]. $N$-glycosylation is a posttranslational modification that plays an important role in the stability, solubility and localization of proteins to the cell surface [117]. $N$-glycosylated proteins can be enriched from biological samples using chemoproteomic- and lectin-based approaches [118, 119]. As $N$-glycosylation is highly prevalent among extracellular proteins (including secreted proteins) and is not present on several high-abundance blood proteins (i.e., albumin), the $\mathrm{N}$-glycoproteome represents a clinically relevant sub-proteome for liquid biomarker discovery. Sinha et al. [120] recently devised an integrated $\mathrm{N}$-glycoproteomics-based approach for detecting biomarkers of HGSC relapse. $N$-glycosylated peptides were enriched from the sera and tumors from recurrent HGSC patient-derived xenograft (PDX) mice and from sera of non-engrafted mice. Species mapping was used to distinguish between peptides of human (tumor) and mouse (stroma) origin, and comparative analysis was used to select a set of candidate markers. Subsequently, PRM was used to quantify the candidates in longitudinal HGSC patient serum samples, revealing four candidates that demonstrated an earlier rise between the remission and the recurrence time points than CA125. Although large-scale clinical validation of the markers is warranted, this study is a proof-of-concept for the use of $\mathrm{N}$-glycoproteomics and PDX models in serum protein biomarker discovery for HGSC.

\section{Glycans, lipids and metabolites}

Posttranslational modifications and metabolic processes are important determinants of cellular signaling and modulating phenotypes. As such, these molecular classes also represent promising candidates for bloodbased biomarker discovery. Considering that aberrant glycosylation occurs during malignant transformation [121], one such approach consists of profiling differences in glycan structures on glycoproteins. Biskup et al. [122] used MS to compare the serum $\mathrm{N}$-glycome profiles between serous EOC patients and healthy women. This glycomics study revealed a marker panel comprising 11 differentially abundant glycans that demonstrated an improved specificity for distinguishing patients from healthy controls compared to CA125. Moreover, as metabolic alterations have been implicated in tumorigenesis [123], metabolomics and lipidomics have emerged as potential avenues for biomarker discovery. Zhou et al. [124] used MS to examine the metabolite profiles of sera from HGSC patients, women with benign ovarian masses and healthy controls and subsequently developed a machine-learning algorithm for diagnostic classification based on the mass spectrum profiles. Buas et al. [125] performed lipidomics analyses of plasma collected from serous EOC patients and patients with benign ovarian masses. A classification model incorporating CA125 and four lipid metabolites demonstrated an increased diagnostic accuracy compared to CA125 alone. Together, these studies suggest a potential utility for plasma metabolites to aid in the diagnosis of HGSC.

\section{Extracellular vesicles and circulating tumor cells}

Aside from investigating freely circulating molecules in blood, molecular profiling of extracellular vesicles (EVs) and circulating tumor cells (CTCs) are alternative approaches for blood-based biomarker discovery. EVs, such as exosomes, are secreted from most cell types, play a role in intercellular communication and contain molecular content from the cell-of-origin [126]. In a pilot study, Taylor et al. [127] identified eight exosomal miRNAs that demonstrated significantly distinct expression profiles in the sera of serous EOC patients compared to the sera of women with benign disease. These exosomal miRNAs exhibited a similar expression profile in tumor tissue and were not detected in the sera of healthy controls. Recently, Kobayashi et al. [128] used microarrays to profile miRNAs in exosomes 
isolated from the conditioned media of HGSC cell lines and immortalized ovarian surface epithelial cells. A single upregulated miRNA was selected for subsequent quantification in EOC patient sera and was found to be differentially expressed between sera of HGSC patients and sera from non-HGSC patients [128]. This illustrates a potential for noninvasive molecular stratification of EOC. Peng et al. [129] compared the proteomes of serum exosomes from serous EOC patients and tumor tissue, revealing 35 proteins commonly upregulated in comparison with normal samples. These findings suggest that exosomes may be of use for noninvasive molecular tumor examinations.

CTCs are tumor cells that are shed into vasculature and play an important role in metastasis [130]. Although CTCs have been explored as noninvasive tumor markers in the general context of EOC, to the best of our knowledge, there are no published investigations specifically focusing on HGSC to date. Studies have primarily focused on the detection and/or the enumeration of CTCs as potential biomarkers in EOC, yet there have been conflicting reports potentially due to differences in isolation strategies [131-133]. Molecular investigations of CTCs are less prevalent and have traditionally involved the use of qRT-PCR to evaluate the expression of a few specific genes. Zhang et al. [134] examined the expression of six genes that were known to be associated with EOC and demonstrated that EpCAM and ERBB2 expressions in CTCs were correlated with platinum resistance and overall survival. Emerging technologies in microfluidics-based CTC isolation and single-cell molecular analysis present new avenues for high-throughput examinations of individual CTCs. Single-cell RNA sequencing of CTCs has been shown to be promising in understanding clonal resistance and metastasis to potentially inform therapeutic decisions in other cancers $[135,136]$. Furthermore, MS-based workflows have recently been developed to profile the proteomes of CTCs, allowing for another layer of molecular characterization [137, 138]. Although single-cell approaches have yet to be applied to CTCs in HGSC, it is proposed that comprehensive molecular characterization of CTCs can provide noninvasive insights regarding intratumor heterogeneity and aid in patient selection for targeted therapies.

\section{Targeted therapies for HGSC}

Targeted therapies are therapeutic agents that act on specific molecular targets, pathways or aspects of the tumor microenvironment that drive the cancer phenotype, in an effort to reduce harm in normal cells. Contemporary systemic management of EOC has progressed from chemotherapy to combination treatments and frontline targeted therapy, when appropriate. In this section, we review the current application of targeted therapies in HGSC clinical practice and describe high-throughput biological workflows for therapeutic target discovery.

\section{Current clinical use of targeted therapies}

Although there are several emerging therapies under clinical investigation for EOC (e.g., immunotherapies [139] and folate receptor-targeted therapies [140]), we have limited our review on the targeted therapies with the most clinical data and they have been approved for use in the clinic (Fig. 3).

\section{Anti-angiogenic agents}

Angiogenesis is a rate-limiting step in the evolution of cancer [141] and has therefore been studied as a potential target for systemic treatment. Bevacizumab is a monoclonal antibody that targets vascular endothelial growth factor (VEGF) A which is secreted by tumors to induce the formation of new blood vessels [142]. Early studies of bevacizumab have demonstrated improved progression-free (PFS) and overall survival (OS) in colorectal and renal cancer [143, 144]. Two landmark trials that assessed the role of concurrent and maintenance treatment with bevacizumab in EOC were the GOG-0218 (primary endpoint: PFS) and the ICON7 (primary endpoints: PFS and OS) studies [145, 146]. Both trials have shown significant improvements in PFS in the intention-to-treat populations with bevacizumab compared to chemotherapy alone but have failed to improve OS in the overall study population. The clinical significance of a three-month difference in PFS has been debated, and as such, bevacizumab is not universally used in the first-line treatment of EOC. In the ICON7 trial, women with high-risk features (inoperable stage III, suboptimal debulking and stage IV disease) randomized to bevacizumab had a significant improvement in mean OS of 4.8 months [147]. Similarly, a subanalysis of the GOG-0218 study suggested that patients with International Federation of Gynecology and Obstetrics (FIGO) stage IV disease may have an increased survival benefit from bevacizumab [148].

In addition to its utility as a first-line therapy, bevacizumab has proven to be effective in patients with recurrent disease. Clinical trials have revealed significant improvements in PFS when bevacizumab was added compared to chemotherapy alone in both platinum-sensitive [149] and platinum-resistant patients [150]. Similar to primary disease, the use of bevacizumab for recurrent disease was not associated with significant improvement in OS for all participants 


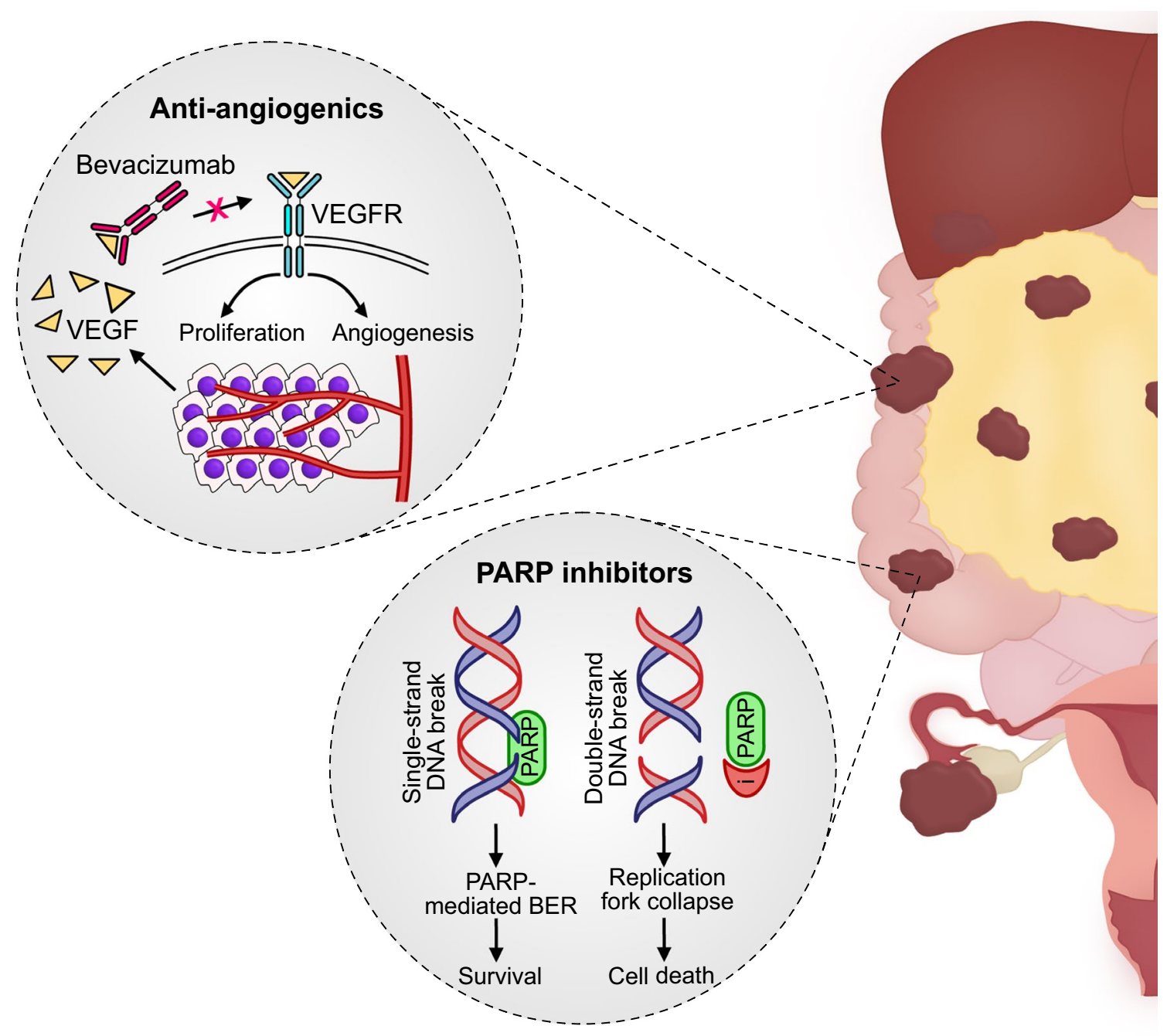

Fig. 3 Current targeted therapies for high-grade serous ovarian cancer. a Anti-angiogenic agents. Cancer cells secrete vascular endothelial growth factor (VEGF) A that binds to vascular endothelial growth factor receptor (VEGFR) to promote angiogenesis and proliferation. Bevacizumab is a monoclonal antibody which inhibits the binding of VEGF to VEGFR, thus hindering angiogenesis and tumor growth. b Poly(ADP-ribose) polymerase (PARP) inhibitors. PARP enzymes mediate base excision repair of DNA single-strand breaks. Inhibition of PARP results in the accumulation of single-strand breaks culminating in DNA double-strand breaks. In cells with homologous repair deficiencies, double-strand breaks are not repaired resulting in replication fork collapse, chromosome instability and cell death. BER base excision repair, PARP poly (ADP-ribose) polymerase, VEGF vascular endothelial growth factor, VEGFR vascular endothelial growth factor receptor

[150,151]. Other anti-angiogenic therapeutics currently under investigation for EOC include pazopanib [152] and nintedanib [153], both of which have shown similar improvements in progression-free survival in clinical trials. Considering the potential severe side effects including hypertension, renal complications, hemorrhage, gastrointestinal perforation and fistula formation, appropriate patient selection and balancing the risks and potential benefits play a pivotal role for anti-angiogenic treatment.

\section{Poly (ADP-ribose) polymerase inhibitors}

PARP-1 was first described in 1966 [154] but its pivotal role for ovarian cancer was only recently discovered [155]. PARP-1 and PARP-2 are enzymes that play a critical role in base excision repair, a repair mechanism for DNA single-strand breaks [156]. The inhibition of PARP results in an accumulation of single-strand breaks, which can lead to double-strand breaks during replication. The double-strand breaks are normally repaired by a process termed 'homologous recombination' [156]. In cancer cells with BRCA1/2 mutations or other HRDs, 
the inhibition of PARP results in a synthetic lethal interaction as the accumulation of double-strand breaks coupled with inadequate repair mechanisms can lead to chromosomal instability, cell cycle arrest and subsequent apoptosis [157]. Olaparib, niraparib and rucaparib are the three PARP inhibitors that are currently FDA-approved for recurrent ovarian cancer after showing consistent improvement in PFS [88, 158-160].

In the SOLO-1 trial, olaparib was tested as a frontline maintenance treatment in women with newly diagnosed FIGO stage III-IV ovarian cancer with germline or somatic BRCA1/2 mutation following cytoreductive surgery and platinum-based chemotherapy. PFS was significantly improved in the olaparib arm compared to placebo and the 3-year progression-free survival was $60.4 \%$ versus $26.9 \%$, HR $0.3(p<0.001)$ [89]. The FDA has subsequently approved olaparib for frontline maintenance treatment in women with platinumresponsive ovarian cancer and $B R C A 1 / 2$ mutation. More recently, the PRIMA trial has shown that niraparib is also effective in the overall population regardless of the HRD status, but the post hoc subanalysis has clearly shown that those patients with $B R C A 1 / 2$ mutations and other HRDs benefited most from maintenance treatment with niraparib [161].

For patients at high risk for recurrence/progression, there is currently a lack of evidence to suggest the superiority of either anti-angiogenic agents or PARP inhibitors over the other. Interim data from the ongoing PAOLA study investigating the combination of PARP inhibitors and bevacizumab suggest a significant benefit in PFS from concurrent use of both agents [153]. Future studies will need to identify patient groups who benefit most from PARP inhibitors, anti-angiogenic treatment, evolving therapeutics such as immunotherapies or a combination of these, while balancing the benefits and added toxicities from combination treatments.

\section{Large-scale discovery of therapeutic targets}

High-throughput experiments can serve as useful means of discovery in targeted therapy development. These large-scale molecular examinations enable the generation of novel hypotheses regarding putative therapeutic targets to help select candidates for further validation. As HGSC is characterized by a lack of discernible drivers (aside from TP53) and extensive heterogeneity, there remains a vast potential for uncovering unanticipated vulnerabilities as novel targeted therapies. Highthroughput experiments for therapeutic target identification can be classified in one of two broad themes: molecular profiling to detect aberrantly expressed molecules in tumors or phenotypic screening to determine the molecules important for cancer cell survival.

\section{Molecular expression profiling}

In molecular expression profiling experiments, highthroughput discovery experiments are performed on one or more molecular classes with the underlying assumption that differences in the expression profile of a molecule, or group of molecules (i.e., those associated with a biological pathway), may inform the understanding of disease pathology. As such, the discovery experiments are typically followed by analyses designed to reveal these differences, including differential expression analyses and ontologically informed pathway analyses. Molecular profiling experiments on patient cohorts enable matching clinical data to molecular phenotype which can be especially apt for identification of candidates for targeted therapies. In one such study, Coscia et al. [162] compared the proteomic profiles of platinum-sensitive and -resistant HGSC patient samples revealing cancer/testes antigen 45 (CT45) expression to be predictive of disease-free survival. By establishing a link between demethylating agents and CT45 expression and by linking CT45 to cytotoxic $\mathrm{T}$ cell engagement, two potential therapeutic strategies can be devised from these findings.

Molecular expression profiling experiments offers the advantage of not requiring a priori information regarding the pathology of the disease. However, a significant hurdle which lays between molecular expression profiling experiments and novel therapeutics is the potential need to develop novel drug compounds. While there are some measures for druggability which can inform therapeutic target selection, drug development remains a costly process with uncertain success [163]. One strategy to mitigate this challenge is to focus on protein classes for which there is a history of therapeutic intervention. For instance, though kinases are often effective targets for cancer therapeutics, established protein kinase therapies have demonstrated limited utility in HGSC. Recognizing this disconnect, Kurimchak et al. [164] profiled the kinome of primary tumors and PDXs to detect differentially expressed kinases in HSGC revealing a potential therapeutic target, MRCKA.

Surface proteins are another especially useful class of molecules as their accessibility renders them favorable therapeutic targets-evidenced by the fact that over 58\% of the known protein targets of FDA-approved drugs are cell surface proteins [165]. However, proteomic workflows which enrich for surface proteins typically require large starting amounts and are therefore not practical or possible for all systems. Despite the challenges associated with surface proteomic workflows, 
the profiling of the cell surface proteins is well suited for identifying targets for repurposing approved therapeutics and development of new therapeutics. Antibody-drug conjugates (ADC), an emerging class of therapeutics for cancer treatment, enable surface proteins to act as potential therapeutic targets independent of their direct connection to disease pathology $[166,167]$. One ADC, IMGN853, which targets folate receptor a, is being evaluated in a phase 3 clinical trial for folate receptorpositive platinum-resistant EOC patients [168]. In this case, although there is some evidence to suggest that targeting folate receptor a alone could have an effect on cancer progression [169], the cytotoxic component of IMGN853 is the maytansinoid compound conjugated to the antibody which targets microtubules.

\section{Phenotypic screening}

Phenotypic screening approaches can be used to identify tumor-specific molecular dependencies as putative targets for therapeutic inhibition. Technological advancements have enabled large-scale molecular perturbations which allow for the functional examination of thousands of molecules in a single experiment. Functional genomic screens entail the use of RNA interference (RNAi) or more recently, clustered regularly interspaced short palindromic repeats (CRISPR)-Cas9 systems coupled with NGS to characterize the genes associated with a phenotype of interest $[170,171]$. In a pan-cancer comparison, Cheung et al. [172] performed genome-wide shRNA knockdown screens in 102 cell lines, including 25 ovarian cancer cell lines revealing 54 genes exclusively essential for ovarian cancer viability and proliferation, underlining the utility of functional genomic approaches to identify lineage-specific dependencies. Functional genomic screening can also be used to identify concurrent therapeutic targets that improve chemosensitivity of existing therapies. Fang et al. [173] performed a genome-wide CRISPR knockout screen in an HGSC cell line treated with olaparib to identify targets that mimic HRD. Based on this screen, the authors were able to characterize a gene whose knockout increased the cytotoxic effects and can potentially extend the clinical utility of PARP inhibitors in HGSC.

In contrast to genome-wide interrogations, functional genomics screens can also be conducted on a subset of experimentally relevant genes. To identify novel synthetic lethal targets in BRCA2 deficient tumors, Mengwasser et al. [174] conducted targeted CRISPR screens in two pairs of isogenic cell lines; one breast cancer pair and one HGSC pair. The isogenic cell lines differed based on the presence of a functional BRCA2 gene and the screen targeted 380 genes that are involved in DNA damage repair. Interestingly, the authors determined two candidates that not only demonstrated synthetic lethality with $B R C A 2$ deficiencies but also BRCA1, as evident through subsequent investigations. The candidates identified in this study represent potential therapeutic targets for $B R C A$-deficient tumors that are resistant to PARP inhibitors. Likewise, Baratta et al. [175] designed an in vivo shRNA screen to assess the depletion of $\sim 800$ genes in xenografts of a human HGSC cell line. The screen revealed several candidates essential for proliferation/survival of HGSC. Through further investigations in patient-derived cell lines, one gene was identified as a potential target for $M Y C N$ overexpressing tumors.

Although functional genomics experiments are advantageous approaches for identifying molecular vulnerabilities for potential inhibition, exploiting these candidates as therapeutic targets can be hindered by the druggability of proteins. The use of high-throughput drug screens is an alternative phenotypic screening approach to identify actionable dependencies. In these experiments, numerous small-molecule compounds, typically with known mechanisms of action, are simultaneously tested against cells to identify novel vulnerabilities. Kenny et al. [176] recently performed a fully robotic screen of $\sim 45,000$ small molecules in an HGSC organotypic model consisting of one of five HGSC cell lines, primary human stromal cells and extracellular matrix components. Subsequent in vitro and in vivo assays identified three compounds that prevent cancer adhesion, proliferation and invasion suggesting that these compounds can be promising therapeutics for ovarian cancer metastasis. Additionally, drug screens can be used to elucidate indirect mechanisms of targeting undruggable cancer proteins. Zeng et al. [177] screened a small molecule library in two HGSC cell lines to identify alternative methods of down-regulating $M Y C$; an oncogene essential when overexpressed in HGSC yet pharmacologically undruggable. This screen revealed a compound that suppressed $M Y C$ expression through simultaneous inhibition of three specific cyclindependent kinases, hence identifying putative targets for $M Y C$ overexpressing HGSC tumors. A caveat associated with drug screens is that target discovery is restricted to those proteins with existing small molecule inhibitors, thus ignoring the potential of other therapeutic classes such as monoclonal antibodies.

\section{Integrated target discovery workflows}

The use of the aforementioned high-throughput experiments is beneficial as initial steps in therapeutic target discovery. Both approaches enable the concurrent screening of numerous molecules to select potential 
candidates for further validation experiments. However, as technological advancements improve the capabilities of these platforms, these large-scale experiments can identify hundreds of hits and given the time-consuming nature of molecular biology interrogations, it is often not feasible to individually follow up on every single hit. The use of bioinformatic tools that prioritize targets (e.g., SurfaceGenie [178]) or mining publicly available data, such as TCGA and the Genotype-Tissue Expression (GTEx) project, can help further narrow down candidates, yet well-annotated complete data are not always available for all diseases. Hence, an emerging workflow for therapeutic target discovery is the integration of both high-throughput approaches for a multifaceted characterization of candidates. By leveraging the advantages of these two orthogonal approaches, an integrated workflow can produce a refined list of candidates that are both actionable and essential. Medrano et al. [179] conducted genome-wide shRNA screens and cell-surface characterizations of 27 HGSC cell lines in parallel, resulting in the identification of CD151 as a cell-surface protein that demonstrated essentiality in a subset of HGSC cell lines. Subsequently, the authors performed RNA sequencing to molecularly characterize the discrepancies in response to knockout of the candidate. This study identified both a novel therapeutic target and a molecular marker of target sensitivity, highlighting the utility of integrated highthroughput workflows for HGSC target discovery. Similar target discovery pipelines have proven promising in other cancer settings as well. Martinko et al. [180] used MS to evaluate changes in cell surface expression associated with oncogenic KRAS and in parallel, conducted a targeted CRISPR knockdown screen of $\sim 1600$ membrane proteins to functionally characterize the oncogenic KRAS surfaceome. Integration of both datasets resulted in the discovery of CDCP1 as a therapeutic antibody target for KRAS-driven cancer cells. Considering the limited success in translating promising targets into beneficial therapies, comprehensive characterization through both expression profiling and functional analyses early in the discovery process may help ensure the selection of robust targets for therapy development.

\section{Experimental model systems \\ Cell lines}

Ideal experimental models should accurately reflect tumor biology to ensure maximum translational utility. Given the ease of use and accessibility, immortalized human cancer cell lines are the most widely used models for experimental interrogations of HGSC [181]. However, until recently, the majority of cell lines used in HGSC research were poorly characterized with uncertain histopathological origins. To address these ambiguities, Domcke et al. [182] compared copynumber changes, mutations and mRNA expression profiles of 47 EOC cell lines and 316 HGSC tumor samples examined by TCGA. Strikingly, this extensive evaluation concluded that the most frequently used cell lines in HGSC research poorly recapitulated the genomic and transcriptomic features of HGSC tumors and are likely other EOC histopathologies. The authors recommended an alternative set of cell lines that closely resemble HGSC tumors and thus would be more appropriate as in vitro models. A separate proteomic profiling study of 28 EOC cell lines, two immortalized ovarian surface epithelial cell lines, three primary fallopian tube epithelial cell isolates and eight HGSC tumor tissues revealed distinct groups of cell lines [183]. The majority of cell lines reported to likely represent HGSC as per Domcke et al. [182] clustered with the proteomes of HGSC tumors and fallopian tube samples, further confirming a HGSC histopathology. Additional studies have revealed discrepancies amongst the ability of HGSC cell lines to model tumor metastasis and histopathology in vivo when xenografted [184, 185]. Together, these studies illustrate the disconnect between certain model cell lines and HGSC tumors and highlight the importance of informed cell line selection.

Another caveat of in vitro cell line models is the artificial microenvironment invoked by monolayer growth on plastic and the lack of multicellularity. Three-dimensional (3D) culture has emerged as a step toward bridging the gap between in vitro and in vivo experiments. 3D culture more closely resembles the tumor microenvironment by restoring 3D cell-cell and cell-ECM interactions [186, 187]. Moreover, research groups have successfully demonstrated co-culturing with patient-derived fibroblasts [188] and patientderived mesothelial cells [189] in HGSC spheroid models to capture the influence of tumor-stromal cross talk on survival and proliferation. Provided that HGSC disseminates through the release of multicellular aggregates into the peritoneal cavity, 3D organotypic in vitro models have been developed to recapitulate significant events in metastasis and gain insights into tumor biology [190]. Nonadherent 3D models have also been used to investigate cancer stem cell populations enriched in disseminated spheroids which are thought to contribute to chemoresistance in HGSC [191]. The use of organ-specific growth factors to model niche environments has enabled the development of ovarian cancer organoid lines that maintain the genomic and histological features of primary tumors and preserve tumor heterogeneity, highlighting their potential utility for precision medicine research [192]. 


\section{Genetically engineered mouse models}

Genetically engineered mouse models (GEMMs) offer the potential for in vivo tumor investigation. Various molecular biology techniques can be used to introduce genetic modifications in a spatial and temporal manner for in vivo modeling of genetic defects contributing to tumorigenesis [193]. However, the preclinical utility of these models is dependent on the accuracy in recapitulating the histology and pathogenesis of human tumors, an element which has historically proven difficult in the context of HGSC. Given the uncertainty in the site of origin for HGSC, early attempts to develop HGSC GEMMs have focused on targeting the ovarian surface epithelium for genetic manipulations [193]. These models failed to replicate the molecular and clinical features observed in human HGSC tumors. In addition to selecting the correct cell of origin, targeting genes that are relevant to HGSC is also an important consideration when generating GEMMs. Indeed, targeting different combinations of oncogenes and tumor suppressors in the same cell of origin has resulted in different HGSC GEMM phenotypes [194, 195]. Fortunately, as molecular understanding of HGSC biology evolves, so does the ability to correctly model the disease. Targeting HGSC relevant genes, such as TP53, BRCA1, RB1 and $P T E N$, in fallopian tube epithelial cells has resulted in a new generation of HGSC GEMMs [195-197]. These clinically relevant models reproducibly demonstrated the formation of precursor STICs in fallopian tubes and mirrored the aggressive metastatic patterns observed in human HGSCs. Hence, if modeled correctly, GEMMs represent promising options for interrogations of earlystage disease and identifying new therapeutic targets.

\section{Patient-derived xenografts}

PDXs are an alternate approach for in vivo experimental models, in which minced fragments of patient tumors are transplanted into immunodeficient mice [198]. The primary advantage of this experimental system is the ability to perform in vivo interrogations of human tumors. Although PDXs have been successfully generated through various different engraftment locations, orthotopic engraftment is preferred as it results in a physiologically relevant microenvironment [199]. In the context of HGSC, there are two engraftment sites that are considered orthotopic: intrabursal (IB) engraftment and intraperitoneal (IP) engraftment [181]. IB engraftment refers to the injection of tumor cells into the ovarian bursa, which is the fat pad surrounding a murine ovary [200]. As there are anatomic differences between the reproductive systems of mice and humans, the bursa can often hinder the extensive peritoneal metastasis characteristic of advanced human ovarian tumors [200].
Alternatively, IP engraftment consists of injecting the tumor cell suspension directly into the peritoneal cavity, mirroring human abdominal tumor dissemination [120, 200]. In addition to recapitulating tumor pathology, orthotopic HGSC PDXs have also been shown to maintain molecular profiles highly comparable to patient tumors [201] and emulate patient-specific responses to platinum-based therapy [202]. These studies highlight the advantage in using PDXs for identifying novel precision medicine approaches in HGSC as it provides an in vivo opportunity for investigating tumor heterogeneity. Indeed, Weroha et al. [203] developed an orthotopic PDX bank consisting of 241 EOC models that reflected the molecular diversity observed in patients and can be a promising resource to investigate subtype specific biomarkers and therapies. A limitation of PDX models is the inability to evaluate interactions between the tumor and the immune system, an important facet of the tumor microenvironment [198].

\section{Additional considerations for high-throughput studies}

Apart from potential unsuitable experimental models, there are several other factors that can impede the utility of high-throughput studies in precision medicine discovery efforts. Provided that several preanalytical variables (e.g., time to freezing, storage duration, serum vs. plasma etc.) have been shown to influence molecular profiles [116, 204], a lack of standardized sample collection and storage protocols can pose as a challenge and potential source of incompatibility for multisite investigations. Though these variables cannot be retrospectively regulated when using samples from biobanks, information about these preanalytical variables should be collected and examined as possible confounders during data analysis. Another consideration is the need for well-annotated clinical cohorts. HGSC patients with extensive disease preventing optimal surgical debulking are often candidates for neoadjuvant chemotherapy [205]; hence, tumor resection is performed only after a round of therapy. As therapeutics can drive the evolution of tumors, tumor samples from these patients likely reflect a different molecular state than those without prior therapy. It is thus essential when conducting high-throughput investigations of clinical samples to use samples with extensive documentation to account for influences from other clinical variables such as treatment history. Additionally, the unprecedented scale of biological data prompts the need for higher computational infrastructure to effectively store and analyze the immense volume of data. While highthroughput experiments are generally well suited for discovery, ultimately to be of clinical benefit, the findings 
must be integrated into testing regimes which are compatible with the healthcare environment (i.e., costeffective and quick).

\section{Conclusions and future perspectives}

Through applications in molecular subtyping, liquid biopsies, and targeted therapies, advancements in highthroughput technologies have opened new avenues for precision medicine discovery in HGSC. Largescale tumor profiling has provided insights regarding the molecular complexity underlying tumorigenesis. Appreciation of this vast heterogeneity has warranted diverging from the one-size-fits-all approach traditionally used for the management of HGSC and EOC as a whole. The use of genetic testing for $B R C A 1 / 2$ and other HRDs as an indication for the use of PARP inhibitors is an example of the adoption of precision medicine into the clinical management of HGSC, yet the dismal five-year survival rate suggests that more work is still needed. The ability to simultaneously examine thousands of molecules in a single experiment has fueled the discovery of numerous putative tumor markers for liquid biopsies in HGSC. Considering the limited utility of single markers (e.g., CA125) due to tumor heterogeneity, the use of highthroughput tools has enabled the potential for uncovering multimarker panels with improved clinical performance. Large-scale biological experiments have also been utilized for the identification of novel therapeutic targets, and integration of orthogonal approaches can be promising for the detection of actionable vulnerabilities in HGSC.

Despite the alluring potential of high-throughput approaches, failure to appreciate the intricate nature of HGSC biology in research design and experimental models can stifle the translational utility of the findings from these experiments. Considering that HGSC and the other histological subtypes of EOC are distinct diseases characterized by differences in molecular profiles, clinical progression and pathogenesis, EOC is often still examined as a single entity without subtypespecific stratification in preclinical and clinical validation studies, thus acting as a potential confounder of findings. Furthermore, despite increasing evidence indicating fallopian tissue epithelium as the primary tissue of origin for HGSC, many studies continue to use ovarian surface epithelium as 'normal' tissue for comparative experiments, resulting in the potential identification of biologically irrelevant biomarkers and therapeutic targets. As such, it is imperative to incorporate our evolving understanding of HGSC biology in research design to leverage the full potential of emerging highthroughput applications in precision medicine.
Abbreviations

ADC: Antibody-drug conjugate; CA125: Cancer antigen 125; cfDNA: Cell-free DNA; CNA: Copy number alterations; CPTAC: Clinical Proteomic Tumor Analysis Consortium; CRISPR: Clustered regularly interspaced short palindromic repeats; CT: Computed tomography; CTC: Circulating tumor cell; ctDNA: Circulating tumor DNA; EOC: Epithelial ovarian cancer; EV: Extracellular vesicle; FIGO: International Federation of Gynecology and Obstetrics; GEMM: Genetically engineered mouse model; GTEx: Genotype-Tissue Expression; HE4: Human epididymis protein 4; HGSC: High-grade serous carcinoma; HRD: Homologous repair deficiency; IB: Intrabursal; IOTA: International Ovarian Tumor Analysis; IP: Intraperitoneal; LGSC: Low-grade serous carcinoma; miRNA: MicroRNA; MRM: Multiple reaction monitoring; MS: Mass spectrometry; NGS: Next-generation sequencing; OS: Overall survival; PARP: Poly(ADP-ribose) polymerase; PCR: Polymerase chain reaction; PDS: Primary debulking surgery; PDX: Patient-derived xenograft; PFS: Progression-free survival; PRM: Parallel reaction monitoring; RMI: Risk of Malignancy Index; RNAi: RNA interference; ROMA: Risk of Malignancy Algorithm; STIC: Serous tubal intraepithelial carcinoma; TCGA: The Cancer Genome Atlas; VEGF: Vascular endothelial growth factor.

\section{Acknowledgements}

The authors thank all members of the Bernardini and Kislinger laboratories for helpful suggestions.

\section{Authors' contributions}

$M G, C W$ and $M W$ wrote the paper with input from MQB and TK. All authors read and approved the final manuscript.

\section{Funding}

This research was funded in part by the Ontario Ministry of Health and LongTerm Care. MG was supported through a CIHR CGS-M award and the Kristi Piia Callum Memorial Fellowship in Ovarian Cancer Research.

\section{Availability of data and materials}

Not applicable.

\section{Ethics approval and consent to participate}

Not applicable.

Consent for publication

Not applicable.

\section{Competing interests}

The authors declare that they have no competing interests.

\section{Author details \\ ${ }^{1}$ Department of Medical Biophysics, University of Toronto, Toronto, Canada. 2 Division of Gynecologic Oncology, Princess Margaret Cancer Centre, University Health Network, Toronto, Canada. ${ }^{3}$ Department of Obstetrics and Gynecology, Paracelsus Medical University, Salzburg, Austria. ${ }^{4}$ Princess Margaret Cancer Centre, University Health Network, Toronto, Canada. \\ ${ }^{5}$ Department of Obstetrics and Gynaecology, University of Toronto, Toronto, Canada.}

Received: 3 August 2020 Accepted: 28 September 2020

Published online: 09 October 2020

\footnotetext{
References

1. Siegel RL, Miller KD, Jemal A. Cancer statistics, 2020. CA Cancer J Clin. 2020;70(1):7-30.

2. Canadian Government. Canadian Cancer Statistics 2019. https://cance r.ca/Canadian-Cancer-Statistics-2019-EN

3. European Commission. ECIS—European Cancer Information. ecis.jrc. ec.europa.eu

4. Lheureux S, Braunstein M, Oza AM. Epithelial ovarian cancer: evolution of management in the era of precision medicine. CA Cancer J Clin. 2019;68(10):7-25.
} 
5. Shih I-M, Kurman RJ. Ovarian tumorigenesis. Am J Pathol. 2004;164(5):1511-8.

6. Pearce CL, Templeman C, Rossing MA, Lee A, Near AM, Webb PM, et al. Association between endometriosis and risk of histological subtypes of ovarian cancer: a pooled analysis of case-control studies. Lancet Oncol. 2012;13(4):385-94.

7. Kurman RJ, Shih I-M. The dualistic model of ovarian carcinogenesis: revisited, revised, and expanded. Am J Pathol. 2016;186(4):733-47.

8. Kurman RJ. Origin and molecular pathogenesis of ovarian high-grade serous carcinoma. Ann Oncol. 2013;24:x16-21.

9. Jacobs IJ, Menon U, Ryan A, Gentry-Maharaj A, Burnell M, Kalsi JK, et al. Ovarian cancer screening and mortality in the UK Collaborative Trial of Ovarian Cancer Screening (UKCTOCS): a randomised controlled trial. The Lancet. 2016;387(10022):945-56.

10. Menon U, Karpinskyj C, Gentry-Maharaj A. Ovarian cancer prevention and screening. Obstet Gynecol. 2018;131(5):909-27.

11. Torre LA, Trabert B, DeSantis CE, Miller KD, Samimi G, Runowicz CD, et al. Ovarian cancer statistics, 2018. CA Cancer J Clin. 2018:68(4):284-96.

12. Goff BA, Mandel LS, Melancon CH, Muntz HG. Frequency of symptoms of ovarian cancer in women presenting to primary care clinics. JAMA. 2004;291(22):2705-12

13. Lheureux S, Gourley C, Vergote I, Oza AM. Epithelial ovarian cancer. The Lancet. 2019;393(10177):1240-53.

14. Kehoe S, Hook J, Nankivell M, Jayson GC, Kitchener H, Lopes T, et al. Primary chemotherapy versus primary surgery for newly diagnosed advanced ovarian cancer (CHORUS): an open-label, randomised, controlled, non-inferiority trial. The Lancet. 2015:386(9990):249-57.

15. Vergote I, Tropé CG, Amant F, Kristensen GB, Ehlen T, Johnson N, et al. Neoadjuvant chemotherapy or primary surgery in stage IIIC or IV ovarian cancer. N Engl J Med. 2010;363(10):943-53.

16. Siegel R, Naishadham D, Jemal A. Cancer statistics, 2012. CA Cancer J Clin. 2012;62(1):10-29.

17. The Cancer Genome Atlas Research Network. Integrated genomic analyses of ovarian carcinoma. Nature. 2011;474(7353):609-15.

18. Vang R, Levine DA, Soslow RA, Zaloudek C, Shih I-M, Kurman RJ. Molecular alterations of TP53 are a defining feature of ovarian highgrade serous carcinoma. Int J Gynecol Pathol. 2016;35(1):48-55.

19. Ahmed AA, Etemadmoghadam D, Temple J, Lynch AG, Riad M, Sharma $\mathrm{R}$, et al. Driver mutations in TP53 are ubiquitous in high grade serous carcinoma of the ovary. J Pathol. 2010;221(1):49-56.

20. Labidi-Galy SI, Papp E, Hallberg D, Niknafs N, Adleff V, Noe M, et al. High grade serous ovarian carcinomas originate in the fallopian tube. Nat Commun. 2017:8(1):1-11.

21. Konstantinopoulos PA, Ceccaldi R, Shapiro GI, D'Andrea AD. Homologous recombination deficiency: exploiting the fundamental vulnerability of ovarian cancer. Cancer Discov. 2015;5(11):1137-54.

22. Macintyre G, Goranova TE, De Silva D, Ennis D, Piskorz AM, Eldridge M, et al. Copy number signatures and mutational processes in ovarian carcinoma. Nat Genet. 2018;50(9):1262-70.

23. Tothill RW, Tinker AV, George J, Brown R, Fox SB, Lade S, et al. Novel molecular subtypes of serous and endometrioid ovarian cancer linked to clinical outcome. Clin Cancer Res. 2008;14(16):5198-208.

24. Konecny GE, Wang C, Hamidi H, Winterhoff B, Kalli KR, Dering J, et al. Prognostic and therapeutic relevance of molecular subtypes in highgrade serous ovarian cancer. JNCIJ. 2014;106(10):11-8.

25. Verhaak RGW, Tamayo P, Yang J-Y, Hubbard D, Zhang H, Creighton CJ, et al. Prognostically relevant gene signatures of high-grade serous ovarian carcinoma. J Clin Investig. 2012;365:671-9.

26. Leong HS, Galletta L, Etemadmoghadam D, George J, The Australian Ovarian Cancer Study, Köbel M, et al. Efficient molecular subtype classification of high-grade serous ovarian cancer. J Pathol. 2015;236(3):272-7.

27. Zhang H, Liu T, Zhang Z, Payne SH, Zhang B, McDermott JE, et al. Integrated proteogenomic characterization of human high-grade serous ovarian cancer. Cell. 2016;166(3):755-65.

28. Hoogstraat M, de Pagter MS, Cirkel GA, van Roosmalen MJ, Harkins TT, Duran K, et al. Genomic and transcriptomic plasticity in treatment-naive ovarian cancer. Genome Res. 2014;24(2):200-11.

29. Schwarz RF, Ng CKY, Cooke SL, Newman S, Temple J, Piskorz AM, et al. Spatial and temporal heterogeneity in high-grade serous ovarian cancer: a phylogenetic analysis. PLOS Med. 2015;12(2):e1001789.
30. Bashashati A, Ha G, Tone A, Ding J, Prentice LM, Roth A, et al. Distinct evolutionary trajectories of primary high-grade serous ovarian cancers revealed through spatial mutational profiling. J Pathol. 2013;231(1):21-34.

31. Hunt AL, Bateman NW, Hood BL, Conrads KA, Zhou M, Litzi TJ, et al. Extensive intratumor proteogenomic heterogeneity revealed by multiregion sampling in a high-grade serous ovarian tumor specimen. Cancer Res. 2019;12(2):e1001789-837.

32. McPherson A, Roth A, Laks E, Masud T, Bashashati A, Zhang AW, et al. Divergent modes of clonal spread and intraperitoneal mixing in highgrade serous ovarian cancer. Nat Genet. 2016;48(7):758-67.

33. Masoodi T, Siraj S, Siraj AK, Azam S, Qadri Z, Parvathareddy SK, et al. Genetic heterogeneity and evolutionary history of high-grade ovarian carcinoma and matched distant metastases. Br J Cancer. 2020;122(8):1219-30.

34. Eckert MA, Coscia F, Chryplewicz A, Chang JW, Hernandez KM, Pan $\mathrm{S}$, et al. Proteomics reveals NNMT as a master metabolic regulator of cancer-associated fibroblasts. Nature. 2019;569(7758):723-8.

35. Izar B, Tirosh I, Stover EH, Wakiro I, Cuoco MS, Alter I, et al. A singlecell landscape of high-grade serous ovarian cancer. Nat Med. 2020;26(8):1271-9.

36. Hu Z, Artibani M, Alsaadi A, Wietek N, Morotti M, Shi T, et al. The repertoire of serous ovarian cancer non- genetic heterogeneity revealed by single-cell sequencing of normal fallopian tube epithelial cells. Cancer Cell. 2020;37(2):226-7.

37. Bast RC Jr, Skates S, Lokshin A, Moore RG. Differential diagnosis of a pelvic mass. Int J Gynecol Cancer. 2012;22:S5-8.

38. Giede KC, Kieser K, Dodge J, Rosen B. Who should operate on patients with ovarian cancer? An evidence-based review. Gynecol Oncol. 2005;99(2):447-61.

39. Rustin GJS, Bast RC, Kelloff GJ, Barrett JC, Carter SK, Nisen PD, et al. Use of CA-125 in clinical trial evaluation of new therapeutic drugs for ovarian cancer. Clin Cancer Res. 2004;10(11):3919-26.

40. Fleming ND, Cass I, Walsh CS, Karlan BY, Li AJ. CA125 surveillance increases optimal resectability at secondary cytoreductive surgery for recurrent epithelial ovarian cancer. Gynecol Oncol. 2011;121(2):249-52.

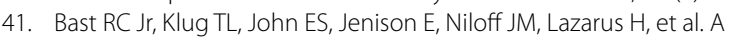
radioimmunoassay using a monoclonal antibody to monitor the course of epithelial ovarian cancer. N Engl J Med. 1983;309(15):883-7.

42. Høgdall EVS, Christensen L, Kjaer SK, Blaakaer J, Kjærbye-Thygesen A, Gayther S, et al. CA125 expression pattern, prognosis and correlation with serum CA125 in ovarian tumor patients. Gynecol Oncol. 2007;104(3):508-15

43. Jacobs I, Bast RC. The CA 125 tumour-associated antigen: a review of the literature. Hum Reprod. 1989;4(1):1-12.

44. Buamah P. Benign conditions associated with raised serum CA-125 concentration. J Surg Oncol. 2000;75(4):264-5.

45. Nossov V, Amneus M, Su F, Lang J, Janco JMT, Reddy ST, et al. The early detection of ovarian cancer: from traditional methods to proteomics. Can we really do better than serum CA-125? Am J Obstet Gynecol. 2008;199(3):215-23.

46. Jacobs I, Oram D, Fairbanks J, Turner J, Frost C, Grudzinskas JG. A risk of malignancy index incorporating CA 125, ultrasound and menopausal status for the accurate preoperative diagnosis of ovarian cancer. $\mathrm{Br} J$ Obstet Gynaecol. 1990;97(10):922-9.

47. Van Calster B, Van Hoorde K, Valentin L, Testa AC, Fischerova D, Van Holsbeke $C$, et al. Evaluating the risk of ovarian cancer before surgery using the ADNEX model to differentiate between benign, borderline, early and advanced stage invasive, and secondary metastatic tumours: prospective multicentre diagnostic study. BMJ. 2014;349:95920.

48. Pinsky PF, Yu K, Kramer BS, Black A, Buys SS, Partridge E, et al. Extended mortality results for ovarian cancer screening in the PLCO trial with median 15 years follow-up. Gynecol Oncol. 2016;143(2):270-5.

49. Rustin GJS, Nelstrop AE, Tuxen MK, Lambert HE. Defining progression of ovarian carcinoma during follow-up according to CA 125: a North Thames Ovary Group study. Ann Oncol. 1996;7(4):361-4.

50. Rustin GJ, Marples M, Nelstrop AE, Mahmoudi M, Meyer T. Use of CA-125 to define progression of ovarian cancer in patients with persistently elevated levels. J Clin Oncol. 2001;19(20):4054-7.

51. Santillan A, Garg R, Zahurak ML, Gardner GJ, Giuntoli RL II, Armstrong DK, et al. Risk of epithelial ovarian cancer recurrence in patients with 
rising serum CA-125 levels within the normal range. J Clin Oncol. 2005;23(36):9338-43.

52. Wilder JL, Pavlik E, Straughn JM, Kirby T, Higgins RV, DePriest PD, et al. Clinical implications of a rising serum CA-125 within the normal range in patients with epithelial ovarian cancer: a preliminary investigation Gynecol Oncol. 2003;89(2):233-5.

53. Rustin GJ, van der Burg ME, Griffin CL, Guthrie D, Lamont A, Jayson GC, et al. Early versus delayed treatment of relapsed ovarian cancer (MRC OV05/EORTC 55955): a randomised trial. The Lancet. 2010;376(9747):1155-63.

54. Niloff JM, Bast RC Jr, Schaetzl EM, Knapp RC. Predictive value of CA 125 antigen levels in second-look procedures for ovarian cancer. Am J Obstet Gynecol. 1985;151(7):981-6.

55. Aravantinos D, Michalas S, Papazefkos V, Christoforaki M, Stypsaneli A, Vlachos $G$, et al. Predictive values of CA 125 antigen levels and $C T$ scan in second-look procedures for ovarian cancer. Eur J Obstet Gynecol Reprod Biol. 1990;37(3):265-70.

56. Drapkin R, von Horsten HH, Lin Y, Mok SC, Crum CP, Welch WR, et al. Human epididymis protein 4 (HE4) is a secreted glycoprotein that is overexpressed by serous and endometrioid ovarian carcinomas. Cancer Res. 2005;65(6):2162-9.

57. Hellström I, Raycraft J, Hayden-Ledbetter M, Ledbetter JA, Schummer M McIntosh M, et al. The HE4 (WFDC2) protein is a biomarker for ovarian carcinoma. Can Res. 2003;63(13):3695-700.

58. Moore RG, Brown AK, Miller MC, Skates S, Allard WJ, Verch T, et al. The use of multiple novel tumor biomarkers for the detection of ovarian carcinoma in patients with a pelvic mass. Gynecol Oncol. 2008;108(2):402-8.

59. Abdel-Azeez HA, Labib HA, Sharaf SM, Refai AN. HE4 and mesothelin: novel biomarkers of ovarian carcinoma in patients with pelvic masses. Asian Pac J Cancer Prev. 2010;11(1):111-6.

60. Holcomb K, Vucetic Z, Miller MC, Knapp RC. Human epididymis protein 4 offers superior specificity in the differentiation of benign and malignant adnexal masses in premenopausal women. YMOB. 2010:205(4):358.e1-358.e6

61. Yanaranop M, Anakrat V, Siricharoenthai S, Nakrangsee S, Thinkhamrop B. Is the risk of ovarian malignancy algorithm better than other tests for predicting ovarian malignancy in women with pelvic masses? Gynecol Obstet Investig. 2017;82(1):47-53.

62. Huhtinen K, Suvitie P, Hiissa J, Junnila J, Huvila J, Kujari H, et al. Serum HE4 concentration differentiates malignant ovarian tumours from ovarian endometriotic cysts. Br J Cancer. 2009;100(8):1315-9.

63. Rosen DG, Wang L, Atkinson JN, Yu Y, Lu KH, Diamandis EP, et al. Potential markers that complement expression of CA125 in epithelial ovarian cancer. Gynecol Oncol. 2005;99(2):267-77.

64. Plotti F, Capriglione S, Terranova C, Montera R, Aloisi A, Damiani P, et al. Does HE4 have a role as biomarker in the recurrence of ovarian cancer? Tumor Biol. 2012;33(6):2117-23.

65. Anastasi E, Giovanna Marchei G, Viggiani V, Gennarini G, Frati L, Reale MG. HE4: a new potential early biomarker for the recurrence of ovarian cancer. Tumor Biol. 2010;31(2):113-9.

66. Schummer M, Drescher C, Forrest R, Gough S, Thorpe J, Hellström I, et al. Evaluation of ovarian cancer remission markers HE4, MMP7 and Mesothelin by comparison to the established marker CA125. Gynecol Oncol. 2012;125(1):65-9.

67. Plotti F, Guzzo F, Schirò T, Terranova C, De Cicco NC, Montera R, et al. Role of human epididymis protein 4 (HE4) in detecting recurrence in CA125 negative ovarian cancer patients. Int J Gynecol Cancer. 2019;29(4):768-71.

68. Nassir M, Guan J, Luketina H, Siepmann T, Rohr I, Richter R, et al. The role of HE4 for prediction of recurrence in epithelial ovarian cancer patients - results from the OVCAD study. Tumor Biol. 2015;37(3):3009-16.

69. Moore RG, Miller MC, DiSilvestro P, Landrum LM, Gajewski W, Ball $J$, et al. Evaluation of the diagnostic accuracy of the risk of ovarian malignancy algorithm in women with a pelvic mass. Obstet Gynecol. 2011;118(21):280-8

70. Moore RG, McMeekin DS, Brown AK, DiSilvestro P, Miller MC, Allard WJ, et al. A novel multiple marker bioassay utilizing HE4 and CA125 for the prediction of ovarian cancer in patients with a pelvic mass. Gynecol Oncol. 2009;112(1):40-6.
71. Li F, Tie R, Chang K, Wang F, Deng S, Lu W, et al. Does risk for ovarian malignancy algorithm excel human epididymis protein 4 and ca125 in predicting epithelial ovarian cancer: a meta-analysis. BMC Cancer. 2012;12(1):1-1.

72. Moore RG, Jabre-Raughley M, Brown AK, Robison KM, Miller MC, Allard WJ, et al. Comparison of a novel multiple marker assay vs the Risk of Malignancy Index for the prediction of epithelial ovarian cancer in patients with a pelvic mass. Am J Obstet Gynecol. 2010;203(3):228. e1-228.e6.

73. Lennox GK, Eiriksson LR, Reade CJ, Leung F, Mojtahedi G, Atenafu EG, et al. Effectiveness of the risk of malignancy index and the risk of ovarian malignancy algorithm in a cohort of women with ovarian cancer: does histotype and stage matter? Int J Gynecol Cancer. 2015;25(5):809.

74. Zhang Z, Chan DW. The road from discovery to clinical diagnostics: lessons learned from the first FDA-Cleared in vitro diagnostic multivariate index assay of proteomic biomarkers. Cancer Epidemiol Biomark Prev. 2010;19(12):2995-9.

75. Ueland FR, Desimone CP, Seamon LG, Miller RA, Goodrich S, Podzielinski I, et al. Effectiveness of a multivariate index assay in the preoperative assessment of ovarian tumors. Obstet Gynecol. 2011;117(6):1289-97.

76. Miller RW, Smith A, Desimone CP, Seamon L, Goodrich S, Podzielinski I, et al. Performance of the American College of Obstetricians and Gynecologists' ovarian tumor referral guidelines with a multivariate index assay. Obstet Gynecol. 2011;117(6):1298-306.

77. Bristow RE, Smith A, Zhang Z, Chan DW, Crutcher G, Fung ET, et al. Ovarian malignancy risk stratification of the adnexal mass using a multivariate index assay. Gynecol Oncol. 2013;128(2):252-9.

78. Coleman RL, Herzog TJ, Chan DW, Munroe DG, Pappas TC, Smith $A$, et al. Validation of a second-generation multivariate index assay for\&nbsp;malignancy risk of adnexal masses. YMOB. 2016;215(1):82. e1-82.e11.

79. Walsh T, Casadei S, Lee MK, Pennil CC, Nord AS, Thornton AM, et al. Mutations in 12 genes for inherited ovarian, fallopian tube, and peritoneal carcinoma identified by massively parallel sequencing. Proc Natl Acad Sci USA. 2011;108(44):18032.

80. Chen S, Parmigiani G. Meta-analysis of BRCA1 and BRCA2 penetrance. J Clin Oncol. 2007;25(11):1329-33.

81. Mavaddat N, Peock S, Frost D, Ellis S, Platte R, Fineberg E, et al. Cancer risks for BRCA1 and BRCA2 mutation carriers: results from prospective analysis of EMBRACE. JNCIJ. 2013;105(11):812-22.

82. Kuchenbaecker KB, Hopper JL, Barnes DR, Phillips K-A, Mooij TM, RoosBlom M-J, et al. Risks of breast, ovarian, and contralateral breast cancer for BRCA1 and BRCA2 mutation carriers. JAMA. 2017;317(23):2402-16.

83. Lancaster JM, Powell CB, Chen L-M, Richardson DL. Society of gynecologic oncology statement on risk assessment for inherited gynecologic cancer predispositions. Gynecol Oncol. 2015;136(1):3-7.

84. Scheuer L, Kauff N, Robson M, Kelly B, Barakat R, Satagopan J, et al. Outcome of preventive surgery and screening for breast and ovarian cancer in BRCA mutation carriers. J Clin Oncol. 2002;20(5):1260-8.

85. Kauff ND, Satagopan JM, Robson ME, Scheuer L, Hensley M, Hudis CA, et al. Risk-reducing salpingo-oophorectomy in women with a BRCA1 or BRCA2 mutation. N Engl J Med. 2002;346(21):1609-15.

86. Alsop K, Fereday S, Meldrum C, deFazio A, Emmanuel C, George J, et al. BRCA mutation frequency and patterns of treatment response in BRCA mutation-positive women with ovarian cancer: a report from the Australian Ovarian Cancer Study Group. J Clin Oncol. 2012;30(21):2654-63.

87. Lesnock JL, Darcy KM, Tian C, Deloia JA, Thrall MM, Zahn C, et al. BRCA1 expression and improved survival in ovarian cancer patients treated with intraperitoneal cisplatin and paclitaxel: a Gynecologic Oncology Group Study. Br J Cancer. 2013;108(6):1231-7.

88. Coleman RL, Oza AM, Lorusso D, Aghajanian C, Oaknin A, Dean A, et al. Rucaparib maintenance treatment for recurrent ovarian carcinoma after response to platinum therapy (ARIEL3): a randomised, double-blind, placebo-controlled, phase 3 trial. The Lancet. 2017;390(10106):1949-61.

89. Moore K, Colombo N, Scambia G, Kim B-G, Oaknin A, Friedlander M, et al. Maintenance olaparib in patients with newly diagnosed advanced ovarian cancer. N Engl J Med. 2018;379(26):2495-505.

90. Matulonis UA, Penson RT, Domchek SM, Kaufman B, ShapiraFrommer R, Audeh MW, et al. Olaparib monotherapy in patients with advanced relapsed ovarian cancer and a germline BRCA1/2 
mutation: a multistudy analysis of response rates and safety. Ann Oncol. 2016;27(6):1013-9.

91. Oza AM, Tinker AV, Oaknin A, Shapira-Frommer R, McNeish IA, Swisher EM, et al. Antitumor activity and safety of the PARP inhibitor rucaparib in patients with high-grade ovarian carcinoma and a germline or somatic BRCA1 or BRCA2 mutation: integrated analysis of data from Study 10 and ARIEL2. Gynecol Oncol. 2017;147(2):267-75.

92. Pepe MS, Etzioni R, Feng Z, Potter JD, Thompson ML, Thornquist M, et al. Phases of biomarker development for early detection of cancer. JNCIJ. 2001;93(14):1054-61.

93. Wan JCM, Massie C, Garcia-Corbacho J, Mouliere F, Brenton JD, Caldas C, et al. Liquid biopsies come of age: towards implementation of circulating tumour DNA. Nat Rev Cancer. 2017;17(4):223-8.

94. Kamat AA, Sood AK, Dang D, Gershenson DM, Simpson JL, Bischoff FZ. Quantification of total plasma cell-free DNA in ovarian cancer using real-time PCR. Ann N Y Acad Sci. 2006;1075:230-4.

95. Zachariah RR, Schmid S, Buerki N, Radpour R, Holzgreve W, Zhong X. Levels of circulating cell-free nuclear and mitochondrial DNA in benign and malignant ovarian tumors. Obstet Gynecol. 2008;112(4):843-50.

96. Shao X, He Y, Ji M, Chen X, Qi J, Shi W, et al. Quantitative analysis of cellfree DNA in ovarian cancer. Oncol Lett. 2015;10(6):3478-82.

97. Cohen JD, Li L, Wang Y, Thoburn C, Afsari B, Danilova L, et al. Detection and localization of surgically resectable cancers with a multi-analyte blood test. Science. 2018;359(6378):926-30.

98. Parkinson CA, Gale D, Piskorz AM, Biggs H, Hodgkin C, Addley H, et al. Exploratory analysis of TP53 mutations in circulating tumour DNA as biomarkers of treatment response for patients with relapsed highgrade serous ovarian carcinoma: a retrospective study. Mardis ER, editor. PLOS Med. 2016;13(12):e1002198-225.

99. Piskorz A, Lin KK, Morris JA, Mann E, Oza AM, Coleman RL, et al. Feasibility of monitoring response to the PARP inhibitor rucaparib with targeted deep sequencing of circulating tumor DNA (ctDNA) in women with high-grade serous carcinoma on the ARIEL2 trial. J Clin Oncol. 2016;34(15_suppl):5549-5549.

100. Kim YM, Lee SW, Lee YJ, Lee HY, Lee JE, Choi EK. Prospective study of the efficacy and utility of TP53 mutations in circulating tumor DNA as a non-invasive biomarker of treatment response monitoring in patients with high-grade serous ovarian carcinoma. J Gynecol Oncol. 2019;30(3):e32-42.

101. Vitale SR, Groenendijk FH, van Marion R, Beaufort CM, Helmijr JC, Jan Dubbink $H$, et al. TP53 mutations in serum circulating cell-free tumor DNA as longitudinal biomarker for high-grade serous ovarian cancer. Biomolecules. 2020;10(3):415-514.

102. Christie EL, Fereday S, Doig K, Pattnaik S, Dawson S-J, Bowtell DDL. Reversion of BRCA1/2Germline mutations detected in circulating tumor DNA from patients with high-grade serous ovarian cancer. J Clin Oncol. 2017:35(12):1274-80.

103. Bettegowda C, Sausen M, Leary RJ, Kinde I, Wang Y, Agrawal N, et al. Detection of circulating tumor DNA in early- and late-stage human malignancies. Sci Transl Med. 2014;6(224):224ra24-224ra24.

104. Harris FR, Kovtun IV, Smadbeck J, Multinu F, Jatoi A, Kosari F, et al. Quantification of somatic chromosomal rearrangements in circulating cell-free DNA from ovarian cancers. Sci Rep. 2016:6:1-9.

105. Shen SY, Singhania R, Fehringer G, Chakravarthy A, Roehrl MHA, Chadwick $D$, et al. Sensitive tumour detection and classification using plasma cell-free DNA methylomes. Nature. 2018;563(7732):579-83.

106. Widschwendter M, Zikan M, Wahl B, Lempiäinen H, Paprotka T, Evans I, et al. The potential of circulating tumor DNA methylation analysis for the early detection and management of ovarian cancer. Genome Med. 2017:9(1):1-14.

107. Peng $Y$, Croce CM. The role of MicroRNAs in human cancer. Sig Transduct Target Ther. 2016;1 (1):843-9.

108. Li L, Zhu D, Huang L, Zhang J, Bian Z, Chen X, et al. Argonaute 2 complexes selectively protect the circulating MicroRNAs in cell-secreted microvesicles. Starczynowski DT, editor. PLoS ONE. 2012;7(10):e46957-9.

109. Mitchell PS, Parkin RK, Kroh EM, Fritz BR, Wyman SK, Pogosova-Agadjanyan EL, et al. Circulating microRNAs as stable blood-based markers for cancer detection. Proc Natl Acad Sci USA. 2008;105(30):10513-8.

110. Todeschini P, Salviato E, Paracchini L, Ferracin M, Petrillo M, Zanotti $\mathrm{L}$, et al. Circulating miRNA landscape identifies miR-1246 as promising diagnostic biomarker in high-grade serous ovarian carcinoma: a validation across two independent cohorts. Cancer Lett. 2017;388:320-7.

111. Shah JS, Gard GB, Yang J, Maidens J, Valmadre S, Soon PS, et al. Combining serum microRNA and CA-125 as prognostic indicators of preoperative surgical outcome in women with high-grade serous ovarian cancer. Gynecol Oncol. 2018;148(1):181-8.

112. Kuhlmann JD, Chebouti I, Kimmig R, Buderath P, Reuter M, Puppel S-H, et al. Extracellular vesicle-associated miRNAs in ovarian cancer-design of an integrated NGS-based workflow for the identification of bloodbased biomarkers for platinum-resistance. Clin Chem Lab Med (CCLM). 2019;57(7):1053-62.

113. Zhang Z, Bast RC Jr, Yu Y, Li J, Sokoll LJ, Rai AJ, et al. Three biomarkers identified from serum proteomic analysis for the detection of early stage ovarian cancer. Cancer Res. 2004;64(16):5882-90.

114. Rai AJ, Zhang Z, Rosenzweig J, Shih I-M, Pham T, Fung ET, et al. Proteomic approaches to tumor marker discovery. Arch Pathol Lab Med. 2002;126(12):1518-26.

115. Anderson NL, Anderson NG. The human plasma proteome. Mol Cell Proteom. 2002;1 (11):845-67.

116. Ignjatovic V, Geyer PE, Palaniappan KK, Chaaban JE, Omenn GS, Baker MS, et al. Mass spectrometry-based plasma proteomics: considerations from sample collection to achieving translational data. J Proteome Res. 2019;18(12):4085-97.

117. Varki A. Biological roles of glycans. Glycobiology. 2016;27(1):3-49.

118. Tian Y, Zhou Y, Elliott S, Aebersold R, Zhang H. Solid-phase extraction of N-linked glycopeptides. Nat Protoc. 2007;2(2):334-9.

119. Kaji H, Saito H, Yamauchi Y, Shinkawa T, Taoka M, Hirabayashi J, et al. Lectin affinity capture, isotope-coded tagging and mass spectrometry to identify N-linked glycoproteins. Nat Biotechnol. 2003;21 (6):667-72.

120. Sinha $A$, Hussain $A$, Ignatchenko $V$, Ignatchenko $A$, Tang $\mathrm{KH}, \mathrm{Ho}$ VWH, et al. N-glycoproteomics of patient-derived xenografts: a strategy to discover tumor-associated proteins in high-grade serous ovarian cancer. Cell Syst. 2019;8:1-16.

121. Dube DH, Bertozzi CR. Glycans in cancer and inflammationpotential for therapeutics and diagnostics. Nat Rev Drug Discov. 2005:4(6):477-88

122. Biskup K, Braicu El, Sehouli J, Fotopoulou C, Tauber R, Berger M, et al. Serum glycome profiling: a biomarker for diagnosis of ovarian cancer. J Proteome Res. 2013;12(9):4056-63.

123. Warburg $\mathrm{O}$. On the origin of cancer cells. Science. 1956;123(3191):309-14.

124. Zhou M, Guan W, Walker LD, Mezencev R, Benigno BB, Gray A, et al. Rapid mass spectrometric metabolic profiling of blood sera detects ovarian cancer with high accuracy. Cancer Epidemiol Biomark Prev. 2010;19(9):2262-71.

125. Buas MF, Gu H, Djukovic D, Zhu J, Drescher CW, Urban N, et al. Identification of novel candidate plasma metabolite biomarkers for distinguishing serous ovarian carcinoma and benign serous ovarian tumors. Gynecol Oncol. 2016;140(1):138-44.

126. Tetta C, Ghigo E, Silengo L, Deregibus MC, Camussi G. Extracellular vesicles as an emerging mechanism of cell-to-cell communication. Endocrine. 2012;44(1):11-9.

127. Taylor DD, Gercel-Taylor C. MicroRNA signatures of tumor-derived exosomes as diagnostic biomarkers of ovarian cancer. Gynecol Oncol. 2008:110(1):13-21.

128. Kobayashi M, Sawada K, Nakamura K, Yoshimura A, Miyamoto M, Shimizu A, et al. Exosomal miR-1290 is a potential biomarker of highgrade serous ovarian carcinoma and can discriminate patients from those with malignancies of other histological types. J Ovarian Res. 2018;11(1):1-10.

129. Peng P, Zhang W, Cao D, Yang J, Shen K. The proteomic comparison of peripheral circulation-derived exosomes from the epithelial ovarian carcinoma (EOC) patients and non-EOC subjects. Transl Cancer Res. 2019:8(2):452-65.

130. Poudineh M, Sargent EH, Pantel K, Kelley SO. Profiling circulating tumour cells and other biomarkers of invasive cancers. Nat Biomed Eng. 2018;2:1-13.

131. Liu JF, Kindelberger D, Doyle C, Lowe A, Barry WT, Matulonis UA. Predictive value of circulating tumor cells (CTCs) in newly-diagnosed and recurrent ovarian cancer patients. Gynecol Oncol. 2013;131(2):352-6. 
132. Pearl ML, Zhao Q, Yang J, Dong H, Tulley S, Zhang Q, et al. Prognostic analysis of invasive circulating tumor cells (iCTCS) in epithelial ovarian cancer. Gynecol Oncol. 2014;134(3):581-90.

133. Lee M, Kim EJ, Cho Y, Kim S, Chung HH, Park NH, et al. Predictive value of circulating tumor cells (CTCS) captured by microfluidic device in patients with epithelial ovarian cancer. Gynecol Oncol. 2017; 145(2):361-5.

134. Zhang X, Li H, Yu X, Li S, Lei Z, Li C, et al. Analysis of circulating tumor cells in ovarian cancer and their clinical value as a biomarker. Cell Physiol Biochem. 2018;48(5):1983-94.

135. Miyamoto DT, Zheng Y, Wittner BS, Lee RJ, Zhu H, Broderick KT, et al. RNA-Seq of single prostate CTCs implicates noncanonical Wnt signaling in antiandrogen resistance. Science. 2015:349(6254):1351-6.

136. Cheng Y-H, Chen Y-C, Lin E, Brien R, Jung S, Chen Y-T, et al. Hydro-Seq enables contamination-free high-throughput single-cell RNA-sequencing for circulating tumor cells. Nat Commun. 2019;10(1):1-11.

137. Li S, Plouffe BD, Belov AM, Ray S, Wang X, Murthy SK, et al. An integrated platform for isolation, processing, and mass spectrometry-based proteomic profiling of rare cells in whole blood. Mol Cell Proteom. 2015;14(6):1672-83

138. Zhu Y, Podolak J, Zhao R, Shukla AK, Moore RJ, Thomas GV, et al. Proteome profiling of 1 to 5 spiked circulating tumor cells isolated from whole blood using immunodensity enrichment, laser capture microdissection, nanodroplet sample processing, and ultrasensitive nanoLC-MS Anal Chem. 2018;90(20):11756-9.

139. Ghisoni E, Imbimbo M, Zimmermann S, Valabrega G. Ovarian cancer immunotherapy: turning up the heat. IJMS. 2019;20(12):2927.

140. Lutz RJ. Targeting the folate receptor for the treatment of ovarian cancer. Transl Cancer Res. 2015;4(1):118-26.

141. Dvorak HF, Brown LF, Detmar M, Dvorak AM. Vascular permeability factor/vascular endothelial growth factor, microvascular hyperpermeability, and angiogenesis. Am J Pathol. 1995;146(5):1029-39.

142. Ferrara N, Hillan KJ, Gerber H-P, Novotny W. Discovery and development of bevacizumab, an anti-VEGF antibody for treating cancer. Nat Rev Drug Discov. 2004;3(5):391-400.

143. Hurwitz H, Fehrenbacher L, Novotny W, Cartwright T, Hainsworth J, Heim W, et al. Bevacizumab plus irinotecan, fluorouracil, and leucovorin for metastatic colorectal cancer. N Engl J Med. 2004;350(23):2335-42.

144. Atkins MB. Management of advanced renal cancer. Kidney Int. 2005;67(5):2069-82

145. Burger RA, Brady MF, Bookman MA, Fleming GF, Monk BJ, Huang $H$, et al. Incorporation of bevacizumab in the primary treatment of ovarian cancer. N Engl J Med. 2011;365(26):2473-83.

146. Perren TJ, Swart AM, Pfisterer J, Ledermann JA, Pujade-Lauraine E, Kristensen $\mathrm{G}$, et al. A phase 3 trial of bevacizumab in ovarian cancer. $\mathrm{N}$ Engl J Med. 2011;365(26):2484-96.

147. Oza AM, Cook AD, Pfisterer J, Embleton A, Ledermann JA, Pujade-Lauraine $\mathrm{E}$, et al. Standard chemotherapy with or without bevacizumab for women with newly diagnosed ovarian cancer (ICON7): overall survival results of a phase 3 randomised trial. Lancet Oncol. 2015;16(8):928-36.

148. Tewari KS, Burger RA, Enserro D, Norquist BM, Swisher EM, Brady MF, et al. Final overall survival of a randomized trial of bevacizumab for primary treatment of ovarian cancer. J Clin Oncol. 2019;37(26):2317-28.

149. Aghajanian C, Blank SV, Goff BA, Judson PL, Teneriello MG, Husain A, et al. OCEANS: a randomized, double-blind, placebo-controlled phase III trial of chemotherapy with or without bevacizumab in patients with platinum-sensitive recurrent epithelial ovarian, primary peritoneal, or fallopian tube cancer. J Clin Oncol. 2012;30(17):2039-45.

150. Pujade-Lauraine E, Hilpert F, Weber B, Reuss A, Poveda A, Kristensen $G$, et al. Bevacizumab combined with chemotherapy for platinumresistant recurrent ovarian cancer: the AURELIA open-label randomized phase III trial. J Clin Oncol. 2014;32(13):1302-8.

151. Aghajanian C, Goff B, Nycum LR, Wang YV, Husain A, Blank SV. Final overall survival and safety analysis of OCEANS, a phase 3 trial of chemotherapy with or without bevacizumab in patients with platinumsensitive recurrent ovarian cancer. Gynecol Oncol. 2015:139(1):10-6.

152. du Bois A, Floquet A, Kim J-W, Rau J, del Campo JM, Friedlander M, et al. Incorporation of Pazopanib in Maintenance Therapy of Ovarian Cancer. J Clin Oncol. 2014;32(30):3374-82

153. Ray Coquard I, Cibula D, Mirza MR, Reuss A, Ricci C, Colombo N, et al. Final results from GCIG/ENGOT/AGO-OVAR 12, a randomised placebo-controlled phase III trial of nintedanib combined with chemotherapy for newly diagnosed advanced ovarian cancer. Int J Cancer. 2019;146(2):439-48.

154. Chambon P, Weill JD, Doly J, Strosser MT, Mandel P. On the formation of a novel adenylic compound by enzymatic extracts of liver nuclei. Biochem Biophys Res Commun. 1966;25(6):638-43.

155. Kraus WL. PARPs and ADP-ribosylation: 50 years ... and counting. Mol Cell. 2015;58(6):902-10.

156. Helleday T, Petermann E, Lundin C, Hodgson B, Sharma RA. DNA repair pathways as targets for cancer therapy. Nat Rev Cancer. 2008;8(3):193-204.

157. Farmer H, McCabe N, Lord CJ, Tutt ANJ, Johnson DA, Richardson TB, et al. Targeting the DNA repair defect in BRCA mutant cells as a therapeutic strategy. Nature. 2005;434(7035):917-21.

158. Ledermann J, Harter P, Gourley C, Friedlander M, Vergote I, Rustin G, et al. Olaparib maintenance therapy in platinum-sensitive relapsed ovarian cancer. N Engl J Med. 2012;366(15):1382-92.

159. Pujade-Lauraine E, Ledermann JA, Selle F, Gebski V, Penson RT, Oza AM, et al. Olaparib tablets as maintenance therapy in patients with platinum-sensitive, relapsed ovarian cancer and a BRCA1/2 mutation (SOLO2/ENGOT-Ov21): a double-blind, randomised, placebo-controlled, phase 3 trial. Lancet Oncol. 2017;18(9):1274-84.

160. Mirza MR, Monk BJ, Herrstedt J, Oza AM, Mahner S, Redondo A, et al. Niraparib maintenance therapy in platinum-sensitive, recurrent ovarian cancer. N Engl J Med. 2016;375(22):2154-64.

161. González Martin A, Pothuri B, Vergote I, DePont CR, Graybill W, Mirza $M R$, et al. Niraparib in patients with newly diagnosed advanced ovarian cancer. N Engl J Med. 2019;381(25):2391-402.

162. Coscia F, Lengyel E, Duraiswamy J, Ashcroft B, Bassani-Sternberg M, Wierer M, et al. Multi-level proteomics identifies CT45 as a chemosensitivity mediator and immunotherapy target in ovarian cancer. Cell. 2018;175(1):159-170.e16.

163. Scannell JW, Blanckley A, Boldon H, Warrington B. Diagnosing the decline in pharmaceutical R\&D efficiency. Nat Rev Drug Discov. 2012;11(3):191-200.

164. Kurimchak AM, Herrera-Montávez C, Brown J, Johnson KJ, Sodi V, Srivastava N, et al. Functional proteomics interrogation of the kinome identifies MRCKA as a therapeutic target in high-grade serous ovarian carcinoma. Sci Signal. 2020;13(619):eaax8238-318.

165. The Human Protein Atlas. The druggable proteome. https://proteinat as.org/humanproteome/tissue/druggable

166. Diamantis N, Banerji U. Antibody-drug conjugates-an emerging class of cancer treatment. Br J Cancer. 2016;114(4):362-7.

167. Peters C, Brown S. Antibody-drug conjugates as novel anti-cancer chemotherapeutics. Biosci Rep. 2015;35(4):126-220.

168. Moore KN, Vergote I, Oaknin A, Colombo N, Banerjee S, Oza A, et al. FORWARD I: a phase III study of mirvetuximab soravtansine versus chemotherapy in platinum-resistant ovarian cancer. Future Oncol. 2018:14(17):1669-78.

169. Sato S, Itamochi H. Profile of farletuzumab and its potential in the treatment of solid tumors. Onco Targets Ther. 2016:9:1181-8.

170. Marcotte R, Brown KR, Suarez F, Sayad A, Karamboulas K, Krzyzanowski PM, et al. Essential gene profiles in breast, pancreatic, and ovarian cancer cells. Cancer Discov. 2012;2(2):172-89.

171. Behan FM, lorio F, Picco G, Alves EGX, Beaver CM, Migliardi G, et al. Prioritization of cancer therapeutic targets using CRISPR-Cas9 screens. Nature. 2019:568(7753):511-6.

172. Cheung HW, Cowley GS, Weir BA, Boehm JS, Rusin S, Scott JA, et al. Systematic investigation of genetic vulnerabilities across cancer cel lines reveals lineage-specific dependencies in ovarian cancer. Proc Natl Acad Sci USA. 2011;108(30):12372-7

173. Fang P, Souza C, Minn K, Chien J. Genome-scale CRISPR knockout screen identifies TIGAR as a modifier of PARP inhibitor sensitivity. Commun Biol. 2019;2:1-16.

174. Mengwasser KE, Adeyemi RO, Leng Y, Choi MY, Clairmont C, D'Andrea $A D$, et al. Genetic screens reveal FEN1 and APEX2 as BRCA2 synthetic lethal targets. Mol Cell. 2019;73(5):885-6.

175. Baratta MG, Schinzel AC, Zwang Y, Bandopadhayay P, Bowman-Colin C, Kutt J, et al. An in-tumor genetic screen reveals that the BET bromodomain protein, BRD4, is a potential therapeutic target in ovarian carcinoma. Proc Natl Acad Sci USA. 2015;112(1):232-7. 
176. Kenny HA, Lal-Nag M, Shen M, Kara B, Nahotko DA, Wroblewski K, et al. Quantitative high-throughput screening using an organotypic model identifies compounds that inhibit ovarian cancer metastasis. Mol Cancer Ther. 2020;19(1):52-62

177. Zeng M, Kwiatkowski NP, Zhang T, Nabet B, Xu M, Liang Y, et al. Targeting MYC dependency in ovarian cancer through inhibition of CDK7 and CDK12/13. Sawyers CL, Levine RL, editors. Elife. 2018;7:e39030.

178. Waas M, Snarrenberg ST, Littrell J, Jones Lipinski RA, Hansen PA, Corbett JA, et al. SurfaceGenie: a web-based application for prioritizing celltype-specific marker candidates. Bioinformatics. 2020;36(11):3447-56.

179. Medrano M, Communal L, Brown KR, Iwanicki M, Normand J, Paterson $J$, et al. Interrogation of functional cell-surface markers identifies CD151 dependency in high-grade serous ovarian cancer. Cell Rep. 2017;18(10):2343-58.

180. Martinko AJ, Truillet C, Julien O, Diaz JE, Horlbeck MA, Whiteley G, et al. Targeting RAS-driven human cancer cells with antibodies to upregulated and essential cell-surface proteins. Elife. 2018;7:e31098.

181. Lengyel E, Burdette JE, Kenny HA, Matei D, Pilrose J, Haluska P, et al. Epithelial ovarian cancer experimental models. Oncogene. 2014;33(28):3619-33.

182. Domcke S, Sinha R, Levine DA, Sander C, Schultz N. Evaluating cell lines as tumour models by comparison of genomic profiles. Nat Commun. 2013:4:1-10.

183. Coscia F, Watters KM, Curtis M, Eckert MA, Chiang CY, Tyanova S, et al. Integrative proteomic profiling of ovarian cancer cell lines reveals precursor cell associated proteins and functional status. Nat Commun. 2016;7:1-14.

184. Mitra AK, Davis DA, Tomar S, Roy L, Gurler H, Xie J, et al. In vivo tumor growth of high-grade serous ovarian cancer cell lines. Gynecol Oncol. 2015;138(2):372-7.

185. Elias KM, Emori MM, Papp E, MacDuffie E, Konecny GE, Velculescu VE, et al. Beyond genomics: critical evaluation of cell line utility for ovarian cancer research. Gynecol Oncol. 2015;139(1):97-103.

186. Pampaloni F, Reynaud EG, Stelzer EHK. The third dimension bridges the gap between cell culture and live tissue. Nat Rev Mol Cell Biol. 2007:8(10):839-45.

187. Tevis KM, Colson YL, Grinstaff MW. Embedded spheroids as models of the cancer microenvironment. Adv Biosyst. 2017;1(10):1700083-133.

188. Hussain A, Voisin V, Poon S, Karamboulas C, Bui NHB, Meens J, et al. Distinct fibroblast functional states drive clinical outcomes in ovarian cancer and are regulated by TCF21. J Exp Med. 2020;217(8):e20191094

189. Hart PC, Kenny HA, GrassI N, Watters KM, Litchfield LM, Coscia F, et al. Mesothelial cell HIF1a expression is metabolically downregulated by metformin to prevent oncogenic tumor-stromal crosstalk. Cell Rep. 2019;29:1-20.

190. Kenny HA, Dogan S, Zillhardt M, Mitra A, Yamada SD, Krausz T, et al. Organotypic models of metastasis: a three-dimensional culture mimicking the human peritoneum and omentum for the study of the early steps of ovarian cancer metastasis. Cancer Treat Res. 2009;149:335-51.

191. Liao J, Qian F, Tchabo N, Mhawech-Fauceglia P, Beck A, Qian Z, et al. Ovarian cancer spheroid cells with stem cell-like properties contribute to tumor generation, metastasis and chemotherapy resistance through hypoxia-resistant metabolism. Orsulic S, editor. PLoS ONE. 2014;9(1):e84941-5013.

192. Kopper O, de Witte CJ, Lõhmussaar K, Valle-Inclan JE, Hami N, Kester $L$, et al. An organoid platform for ovarian cancer captures intra- and interpatient heterogeneity. Nat Med. 2019;25(5):838-49.
193. Jones PM, Drapkin R. Modeling high-grade serous carcinoma: how converging insights into pathogenesis and genetics are driving better experimental platforms. Front Oncol. 2013;3:1-10.

194. Kim J, Coffey DM, Creighton CJ, Yu Z, Hawkins SM, Matzuk MM. Highgrade serous ovarian cancer arises from fallopian tube in a mouse model. Proc Natl Acad Sci USA. 2012;109(10):3921.

195. Zhai Y, Wu R, Kuick R, Sessine MS, Schulman S, Green M, et al. Highgrade serous carcinomas arise in the mouse oviduct via defects linked to the human disease. J Pathol. 2017;243(1):16-25.

196. Perets R, Wyant GA, Muto KW, Bijron JG, Poole BB, Chin KT, et al. Transformation of the fallopian tube secretory epithelium leads to high-grade serous ovarian cancer in Brca;Tp53;Pten models. Cancer Cell. 2013;24(6):751-65.

197. Sherman-Baust CA, Kuhn E, Valle BL, Shih I-M, Kurman RJ, Wang T-L, et al. A genetically engineered ovarian cancer mouse model based on fallopian tube transformation mimics human high-grade serous carcinoma development. J Pathol. 2014;233(3):228-37.

198. Kopetz S, Lemos R, Powis G. The promise of patient-derived xenografts: the best laid plans of mice and men. Clin Cancer Res. 2012;18(19):5160-2.

199. Hoffman RM. Patient-derived orthotopic xenografts: better mimic of metastasis than subcutaneous xenografts. Nat Rev Cancer. 2015;15(8):451-2.

200. Shaw TJ, Senterman MK, Dawson K, Crane CA, Vanderhyden BC. Characterization of intraperitoneal, orthotopic, and metastatic xenograft models of human ovarian cancer. Mol Ther. 2004;10(6):1032-42.

201. Ricci F, Bizzaro F, Cesca M, Guffanti F, Ganzinelli M, Decio A, et al. Patientderived ovarian tumor xenografts recapitulate human clinicopathology and genetic alterations. Cancer Res. 2014;74(23):6980.

202. Topp MD, Hartley L, Cook M, Heong V, Boehm E, McShane L, et al. Molecular correlates of platinum response in human high-grade serous ovarian cancer patient-derived xenografts. Mol Oncol. 2014;8(3):656-68.

203. Weroha SJ, Becker MA, Enderica-Gonzalez S, Harrington SC, Oberg AL, Maurer MJ, et al. Tumorgrafts as in vivo surrogates for women with ovarian cancer. Clin Cancer Res. 2014;20(5):1288-97.

204. Moldovan L, Batte KE, Trgovcich J, Wisler J, Marsh CB, Piper M. Methodological challenges in utilizing miRNAs as circulating biomarkers. J Cell Mol Med. 2014;18(3):371-90.

205. Wright AA, Bohlke K, Armstrong DK, Bookman MA, Cliby WA, Coleman RL, et al. Neoadjuvant chemotherapy for newly diagnosed, advanced ovarian cancer: Society of Gynecologic Oncology and American Society of Clinical Oncology Clinical Practice Guideline. J Clin Oncol. 2016;34(28):3460-73.

\section{Publisher's Note}

Springer Nature remains neutral with regard to jurisdictional claims in published maps and institutional affiliations. 